\title{
NUMERICAL STUDIES OF SHEAR-THINNING DROPLET FORMATION IN A MICROFLUIDIC T-JUNCTION USING TWO-PHASE LEVEL-SET METHOD
}

\author{
Voon-Loong Wong ${ }^{\mathrm{a}, \mathrm{b}, 1}$, Katerina Loizou ${ }^{\mathrm{b}, 2}$, Phei-Li Lau ${ }^{\mathrm{a}}$, Richard S. Graham ${ }^{\mathrm{c}}$, and
} Buddhika N. Hewakandambyb,*

aDepartment of Chemical and Environmental Engineering, University of Nottingham Malaysia Campus, Semenyih 43500, Malaysia.

bDepartment of Chemical and Environmental Engineering, University of Nottingham, Nottingham NG7 2RD, United Kingdom.

'School of Mathematical Sciences, University of Nottingham, Nottingham NG7 2RD, United Kingdom.

\section{ABSTRACT}

A conservative level-set method (LSM) embedded in a computational fluid dynamics (CFD) simulation provides a useful approach for the studying the physics and underlying mechanism in two-phase flow. Detailed two-dimensional (2D) computational microfluidics flow simulations have been carried out to examine systematically the influence of different controlling parameters such as flow rates, viscosities, surface wettability, and interfacial tensions between two immiscible fluids on the non-Newtonian shear-thinning microdroplets generation process. For the twophase flow system that neglects the Marangoni effect, the breakup process of shearthinning microdroplets in cross-flowing immiscible liquids in a microfluidic device with a T-shaped geometry was predicted. Data for the rheological and physical properties of fluids obeying Carreau-Yasuda stress model were empirically obtained to support the computational work. The simulation results show that the relevant control parameters mentioned above have a strong impact on the size of shear-thinning droplets generated. Present computational studies on the role and relative importance of controlling parameters can be established as a conceptual framework

Corresponding author; buddhika.hewakandamby@nottingham.ac.uk

1 Current affiliation: School of Engineering and Physical Sciences, Heriot-Watt University Malaysia Campus,62200 Putrajaya Wilayah Persekutuan, Malaysia.

2. Current affiliation: Department of Chemical Engineering, University College London, London, United Kingdom 
of the non-Newtonian droplet generation process and relevant phenomena for future studies.

Keywords: Carreau-Yasuda; microfluidics; fluid dynamics; level-set simulation; emulsification.

\section{Introduction}

Microfluidic applications are commonly found in coating flows, formation of suspensions, emulsions and foams, heat transfer and fluid flow (Stone et al., 2004; Squires and Quake, 2005; Pipe and McKinley, 2009). Since the start of the micro total analysis system ( $\mu$ TAS), the manipulation of droplets in confined microfluidic channels has been highlighted as a useful technology platform for a broad range of application in many scientific fields such as biology, biomedical studies, chemical and synthesis, and drug delivery. Many of these fields involve handling non-Newtonian fluids characterised by a nonlinear relationship between shear stress and the deformation rate. Previously, most of the studies of droplet formation in microchannels have employed traditional Newtonian-Newtonian systems (Cramer et al., 2004; Garstecki et al., 2006; Anna and Mayer, 2006; Utada et al., 2007, Xu et al., 2008), and little attention has been given to non-Newtonian fluids. However, it has been identified that non-Newtonian microfluidic applications are increasingly taking the centre ground of scientific and commercial interests, demanding research and investigation (Stone et al., 2004).

Diverse forces, including surface tension and viscous forces, govern drop dynamics, and the rheology of the fluid can be important when non-Newtonian fluids are involved (Chhabra and Richardson, 2008). As a general rule, non-Newtonian fluids can be classified into three groups, namely purely viscous fluids, time dependent fluids, and viscoelastic fluids (Chung et al., 2008). Each of the above mentioned non-Newtonian fluid possesses distinct characteristics of their own; however, no single constitutive equation has been established to describe the rheogram of all such fluids. Understanding the dynamical mechanisms of monodispersed micro or nanodroplets formation of non-Newtonian fluids in microfluidic channels is essential to ensure that specific requirements such as, drop size, patterns and productivity are met. 
60 Previously, some researchers have focused on the analysis of the dynamics and deformation of droplets with viscoelastic properties in this area (Husny and CooperWhite 2006; Steinhaus et al., 2007; Arratia et al., 2009; Qiu et al., 2010), yet a thorough understanding of relevant physics behind their creation for other nonNewtonian fluid types is still limited.

Near monodispersed droplets can be generated by a number of methods in microfluidic devices including breakup in co-flowing streams (Cramer et al., 2004, Utada et al., 2007) breakup in cross-flowing stream (Garstecki et al., 2006; Xu et al., 2008; Husny and Cooper-White 2006; Qu et al., 2010), breakup in elongational strained flows (Anna and Mayer, 2006), and breakup in microchannel emulsification (MCE) (Sugiura et al., 2001; Kobayashi et al., 2002; Kobayashi et al., 2005; Van Der Zwan et al., 2009). The T-junction microchannel is one of the most frequently used microfluidic configurations based on the physical mechanism of droplet breakup in a cross-flowing stream. According to this mechanism, viscous shear-stress from the continuous stream of the horizontal channel overcomes surface tension at the liquidliquid interface and pulls off droplets of the dispersed phase entering from the vertical channel. Cross-flow shear in a T-junction microchannel remains one of the most utilised microfluidic configurations, owing to the ease of droplet formation, inherent droplet size controllability, periodicity of droplet formation, and design simplicity (Thorsen et al., 2001; Nisisako et al., 2002; Tice et al., 2004; Garstecki et al., 2005). Those investigations of the emulsification process for non-Newtonian-Newtonian systems found in the literature have been performed using T-shaped microchannels. For instance, Husny and Cooper-White (2006) investigated polymeric droplet creation and the breakup dynamics of low viscosity, elastic polyethylene oxide (PEO) fluids and Newtonian fluids by cross-flow shear. Gu and Liow (2011) investigated the formation of shear-thinning xanthan gum droplets in oil in a T-junction microchannel under different flow conditions. Apart from empirical works, Sang et al. (2009) developed both numerical and analytical models with an interface capturing scheme to explore the influence of the material behaviour of Newtonian, Bingham and powerlaw fluid on the formation of droplets. Qiu et al. (2010) also performed a numerical study of the formation of Newtonian droplets in a continuous phase with shearthinning properties under the cross-flow condition using the volume-of-fluid (VOF) formulation. Most of the previous studies, especially the numerical ones, have 
93 focused on the Newtonian droplets formed in the non-Newtonian phase; however, there have been far fewer attempts to develop a predictive model for the relevant physics, evolution and formation of non-Newtonian droplets in a T-junction microfluidic cell.

Due to the existence of a high surface-to-volume ratio within a micro-scaled device, interfaces between fluids play a significant role in many physical processes such as droplets collision, coalescence and breakup (Christopher et al., 2009). There are two main approaches to represent the interface evolution problem either explicitly or implicitly within the Navier-Stokes (NS) equation discretized on a fixed grid: interface tracking and interface capturing. These methods are widely used in computation of flow problems with moving boundaries and interfaces. Interface tracking of the moving boundary is based on an explicit description of the interface by the computational mesh. Boundary integral (Hou et al., 2001) and front tracking (Tryggvason et al., 2001) methods are examples of interface tracking methods. Interface tracking approaches provide higher numerical accuracy, but their applicability is limited to Stokes flow. In contrast to interface tracking methods, interface motion can be simply obtained by the advection of the corresponding phase function in the interface capturing method. The interface capturing method uses a separate phase function, discretised on the fixed grid, to implicitly represent the interface (Bonometti and Magnaudet, 2007). In implicit approaches, there are three common interface capturing methods that have been developed to solve incompressible two-phase problems. These are the volume-of-fluid (VOF) (Rider and Kothe, 1998), lattice-Boltzmann method (LBM) (Takada et al., 2000), and level-set method (LSM) (Osher and Sethian, 1988). In each of the above reference methods, the main challenge is to accurately advect the phase boundary and correctly compute terms concentrated at the interface, such as interfacial tension. The functional discontinuities of the VOF method present at the interface lead to low accuracy in the computation of the mean curvature, causing errors in the surface tension force. Apart from the VOF method, the variation of interfacial tension has made difficulties in the application of LBM to this problem as this parameter cannot be adjusted independently. However, the above mentioned limitations can be overcome by LSM as it allows any interfacial tension values to be chosen. The level-set method is a simple and versatile method for tracking moving interfaces and shapes. It permits 
numerical computations of such objects involving curves and surface to be performed on a fixed Cartesian grid without having to parameterise them. It is a robust scheme that is relatively easy to implement (Osher and Sethian, 1988). Although the main drawback of classical LS description is the difficulty of ensuring volume conserving advection of fluid phases, a conservative LSM has been devised to overcome this (Olsson and Kreiss, 2005; Sethian, 1999).

This paper describes a numerical study carried out to develop a predictive model for droplet generation of a non-Newtonian fluid at a microfluidic $T$ junction. A conservative LS approach coupled with the Carreau-Yasuda stress model has been adopted in the present computational model. Systematic sets of numerical simulations were carried out to monitor the evolution of droplet size, which turns out to be effectively governed by the physical properties of the tested fluids, flow rates, and viscosity. These key parameters that control the formation of microdroplets of shear-thinning liquids such as sodium carboxymethylcellulose (SCMC) have not previously been examined in detail. In the present study, the continuous phase is a Newtonian fluid with a constant viscosity, whereas the dispersed phase has properties equivalent to shear-thinning SCMC solutions with concentration ranging from $0 \mathrm{wt} \%$ to $1.20 \mathrm{wt} \%$. This provides a realistic means of computing the dynamics of droplet breakup process in a T-junction micro-scaled device when the shear-thinning non-Newtonian effects are present. It should also be noted that within the considered weight percentages, the variation in density is minimal enabling the use of a constant value of $998.2 \mathrm{~kg} / \mathrm{m}^{3}$. Furthermore, the surface tension between the lowest and the highest concentrations are 22.14 and $18.87 \mathrm{mN} / \mathrm{m}$. Therefore a constant value of $20.74 \mathrm{mN} / \mathrm{m}$ is used for all the cases except for the parametric study on the interfacial tension. Both the concentration and the contact angle were treated as independent parameters in this study.

\section{Computational Model}

\subsection{The Conservative Level-Set Method}

The conservative level-set method (LSM) was employed to simulate the filament development and the detachment of the droplets at various operating conditions and fluid properties inside a microchannel T-junction. The conservative LSM proposed by 
157 Olsson and Kreiss (2005) and Olsson et al. (2007) was employed in the present study.

158 The LSM relies on an implicit representation of the interface and the equation of 159 motion is numerically approximated using schemes built from hyperbolic160 conservatives law (Osher and Sethian, 1988). This approach has been used to 161 describe the interface between two immiscible fluids which is defined by the 0.5 162 contour of the level set (phase) function $(\phi)$ assuming the continuous phase $(\phi=0)$ 163 flowing in the main horizontal channel and the dispersed phase $(\phi=1)$ fills a channel 164 orthogonal to the main channel. In this implicit method, the model comprises of the 165 following governing equations.

166 Incompressible Navier-Stokes equation

$$
\rho \frac{\partial \mathbf{u}}{\partial t}+\rho(\mathbf{u} \cdot \nabla) \mathbf{u}=\nabla\left[-p I+\eta\left(\nabla \mathbf{u}+(\nabla \mathbf{u})^{T}\right]+F_{s t}\right.
$$

168 Continuity equation

$169 \nabla \cdot \mathbf{u}=0$

170 Level-set equation

$$
\frac{\partial \phi}{\partial t}+\mathbf{u} \cdot \nabla \phi=\gamma \nabla \cdot\left[\varepsilon \nabla \phi-\phi(1-\phi) \frac{\nabla \phi}{|\nabla \phi|}\right]
$$

172 Symbols $\rho, \eta$, and $\sigma$ denote the density, dynamic viscosity, and the surface tension 173 respectively. The pressure is given by $p$ while $/$ denotes the identity matrix. The $V$ denotes the re-initialization parameter approximates the maximum speed occurring in the computational domain (Sethian, 1999). The $\varepsilon$ denotes an artificial thickness of the interface, which is assumed to be the maximum mesh size in subdomains in the neighbourhood of the interface. In this work, the parameters $y$ and $\varepsilon$ with the value of $0.065 \mathrm{~m} / \mathrm{s}$ and $5.8 \times 10^{-6} \mathrm{~m}$ were selected, based on the maximum flow velocity in 179 microchannel dimensions selected for simulation and optimum mesh size, 180 respectively after a mesh convergence analysis. The surface tension force, $F_{s t}$, acting at the interface between two fluid phases can be calculated by Equation 4 below.

$$
F_{s t}=\sigma k(\phi) \mathbf{n}_{\Gamma} \delta_{s m}(\phi)
$$


183 where $k$ denotes the local interfacial curvature, $\mathbf{n}_{\Gamma}$ is the unit normal vector to the 184 interface pointing into the droplet, and the smeared out Dirac delta function 185 concentrated at the interface between two fluids is given by $\delta_{s m}(\phi)$. These can be 186 expressed as shown below.

187 The interfacial curvature is given by

$188 k(\phi)=-\nabla \cdot \mathbf{n}_{\Gamma}$

189 The delta function is approximated by

$190 \delta_{s m}(\phi)=6|\phi(1-\phi)||\nabla \phi|$

191 Since the surface tension and local interfacial curvature term are easily represented 192 in terms of the level-set function, the LSM can be used to compute the changes in 193 the droplet topology due to the flow. The density and the viscosity of the two fluids at 194 any point can be calculated using the two equations given below:

$195 \rho=\rho_{1}+\left(\rho_{2}-\rho_{1}\right) \phi$

$196 \eta=\eta_{1}+\left(\eta_{2}-\eta_{1}\right) \phi$ where $\rho_{1}$ and $\rho_{2}$ are the densities of continuous phase and dispersed phase, and $\eta_{1}$ and $\eta_{2}$ are the viscosities of continuous phase and dispersed phase.

199 Equations (1) - (8) were solved using the COMSOL Multiphysics (Version 4.3) two phase flow module.

\subsection{Rheological Constitutive Model}

203 To implement the shear thinning non-Newtonian fluids, the Carreau-Yasuda viscosity 204 model has been implemented within the level set formulation. The Carreau-Yasuda 205 constitutive model is defined by the following equation (Eq. (9)) (Carreau, 1972; 206 Yasuda and Cohen, 1981):

$207 \eta(\dot{\gamma})=\eta_{\infty}+\left(\eta_{o}-\eta_{\infty}\right)\left[1+\left(\lambda_{C Y} \dot{\gamma}\right)^{a}\right]^{\frac{n-1}{a}}$ 
208 where $\eta_{0}$ and $\eta_{\infty}$ denote the zero shear viscosity and the infinite shear viscosity respectively whereas $\lambda c y$ denotes the relaxation time and a denotes fitting parameter.

210 The $n$ is the shear-thinning power-law exponent and $\dot{\gamma}$ is the shear rate. We

211 generalize the shear rate $(\dot{\gamma})$ in the Carreau-Yasuda model using the following 212 invariant of the velocity gradient tensor in Cartesian coordinates.

$213 \dot{\gamma}=\sqrt{\frac{1}{2}\left[4\left(\frac{\partial u}{\partial x}\right)^{2}+2\left(\frac{\partial u}{\partial y}+\frac{\partial v}{\partial x}\right)^{2}+4\left(\frac{\partial v}{\partial y}\right)^{2}\right]}$

214 The rheological parameters of Carreau-Yasuda stress model were experimentally 215 determined using a controlled stress rheometer (MC 302, Anton Paar) for all SCMC 216 polymer concentrations. All the measurement data were seen to be fitted well to 217 Carreau-Yasuda model for all SCMC concentrations. Experimentally measured 218 normalised shear-viscosity is plotted against the normalised shear rate for SCMC 219 solutions at various concentrations in Figure 1a. These measurement data are also 220 summarized in Table 1 and used in numerical calculations subsequently. For shear 221 thinning fluids, the flow modifies the viscosity and so the effective viscosity at the flow 222 conditions in a given simulation becomes an important quantity. To aid later 223 interpretation we estimate this by using the wall shear rate in the continuous phase. 224 Figure $1 \mathrm{~b}$ shows the effective viscosity ratio $\left(\lambda_{\eta}=\eta_{d} / \eta_{c}\right)$ against continuous phase flow rate, $Q_{c}$, where we used the downstream wall shear rate in the continuous phase as a 'typical' shear rate and computed the disperse phase viscosity via equation 9. In the present work, a key output quantity is the effective droplet diameter $(d)$. An integration operator was used to find the area corresponds to the dispersed phase where $\phi>0.5$ in order to calculate the effective droplet diameter (Equation 11). 


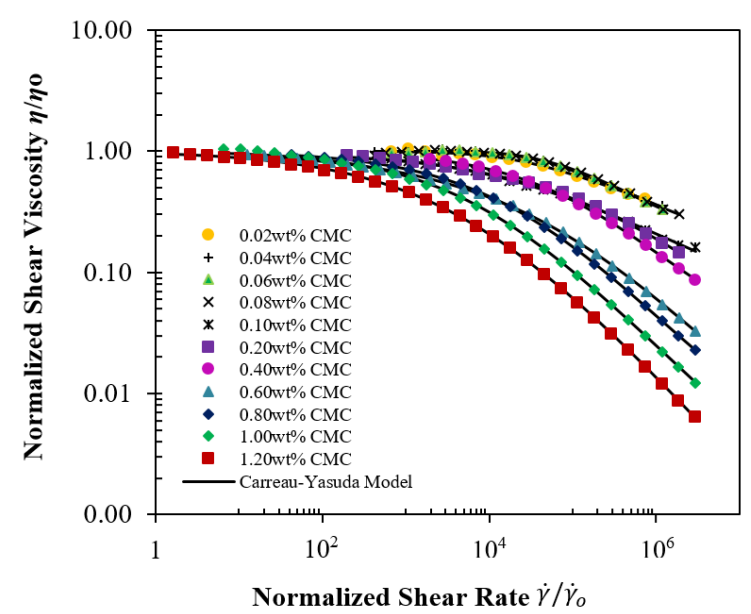

(a)

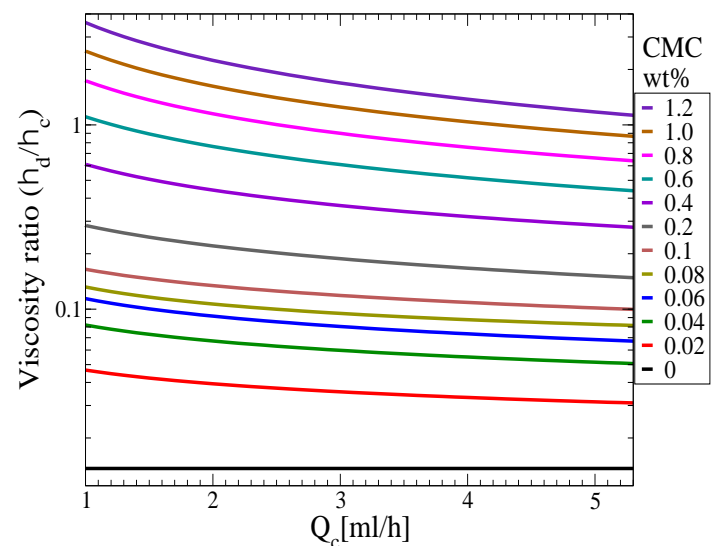

(b)

Figure 1: (a) Normalized shear viscosity plotted against normalized shear rate for a series of SCMC shear-thinning solutions with different concentrations. (b) Effective viscosity ratio against continuous phase flow rate, $Q_{c}$, with the shear-thinning computed from equation 9 using the wall shear rate in the continuous phase.

Table 1: Rheological properties of SCMC polymer solution.

\begin{tabular}{cccccc}
\hline SCMC Solution & \multicolumn{5}{c}{ Carreau-Yasuda Model Constant } \\
\cline { 2 - 6 } wt\% & $\eta_{0}$ (Pa.s) & $\eta_{\infty}$ (Pa.s) & $\lambda c y(s)$ & $n$ & a \\
\hline 0.02 & 0.0070 & 0.0003 & 0.0400 & 0.7121 & 0.9653 \\
0.04 & 0.0121 & 0.0000 & 0.0325 & 0.7102 & 1.6980 \\
0.06 & 0.0171 & 0.0000 & 0.0256 & 0.6775 & 1.3728 \\
0.08 & 0.0195 & 0.0028 & 0.0143 & 0.4886 & 1.1319 \\
0.10 & 0.0420 & 0.0007 & 0.0572 & 0.6242 & 0.4734 \\
0.20 & 0.0742 & 0.0006 & 0.0041 & 0.3528 & 0.3856 \\
0.40 & 0.1946 & 0.0040 & 0.0138 & 0.3157 & 0.5534 \\
0.60 & 0.7995 & 0.0022 & 0.0147 & 0.1995 & 0.3660 \\
0.80 & 1.6469 & 0.0057 & 0.0515 & 0.2444 & 0.4782 \\
1.00 & 4.1143 & 0.0031 & 0.1604 & 0.2869 & 0.5000 \\
1.20 & 10.2644 & 0.0000 & 0.2069 & 0.2297 & 0.4175 \\
\hline
\end{tabular}

\subsection{Geometry and Boundary Conditions}

243 A T-junction geometry with $220 \mu \mathrm{m}\left(w_{c}\right)$ main channel width and a $90 \mu \mathrm{m}\left(w_{d}\right)$ side channel was created and meshed with quadrilaterals elements. These dimensions are selected to match the microchannel geometry created for validation experiments. An entrance thickness $(h)$ of $73.5 \mu \mathrm{m}$ was prescribed in the computational domain in order to define the depth of the microchannel. The continuous phase flows along the 
248 horizontal main channel whereas the dispersed phase is introduced through the narrow side channel. The geometry of the microfluidic T-junction with structured 2D mapped mesh is illustrated in Figure 2. In microfluidics systems, all flow tends to be laminar. The domain boundary of the continuous $\left(\partial \Omega_{1}\right)$ and dispersed phase $\left(\partial \Omega_{2}\right)$ were set up with laminar inflow conditions by defining the volumetric flow rates of

253 continuous phase and dispersed phase.

254

$\partial \Omega_{1}=\left\{\begin{array}{l}u_{x}=u \cdot \mathrm{n}=u_{o} \\ u_{y}=0 \\ h \int_{\alpha \Omega_{1}} u \cdot \mathrm{n} d y=Q_{c}\end{array}\right.$

255

$$
\partial \Omega_{2}=\left\{\begin{array}{l}
u_{x}=0 \\
u_{y}=u \cdot \mathrm{n}=u_{o} \\
h \int_{\partial \Omega_{2}} u \cdot \mathrm{n} d x=Q_{d}
\end{array}\right.
$$

256 where $\mathbf{n}$ is unit vector that has a direction normal to the inlet boundaries, $h$ is the entrance thickness of microchannel, $Q_{c}$ and $Q_{d}$ denote the flow rates of the continuous and dispersed phase flow rates respectively. The domain boundary condition at the outlet $\left(\partial \Omega_{3}\right)$ is set to a pressure with no viscous stress.

$260 P=0 \mathrm{~Pa}$

No-slip boundary condition was applied to all other wall boundaries. The numerical simulations were performed at a time step $\Delta t=2.57 \times 10^{-5}$ seconds, calculated using the Courant-Friedrichs-Lewy (CFL) number. The Courant number is defined as:

$264 \quad C=\frac{\Delta t \cdot U}{\Delta X}$

where $\Delta t$ is the time-step used during simulations, $U$ is the maximum fluid velocity and $\Delta X$ is the computational cell size. A Courant number of 0.25 was used in the current simulations considering as a robust value to maintain the stability of the calculations (Kashid et al., 2010; Schneider et al., 2011). 
Table 2: Mesh convergence analysis for the dependence of effective droplet diameter on the number of quadrilaterals elements.

\begin{tabular}{llll}
\hline $\begin{array}{l}\text { Number of } \\
\text { Elements }\end{array}$ & $\begin{array}{l}\text { Degree of } \\
\text { Freedom }\end{array}$ & $\begin{array}{l}\text { Effective Droplet } \\
\text { Diameter }(\mu \mathrm{m})\end{array}$ & $\begin{array}{l}\text { Relative } \\
\text { Error\% }\end{array}$ \\
\hline \hline 976 & 13590 & 172.46 & 6.960 \\
2072 & 28727 & 162.63 & 1.586 \\
4024 & 54152 & 158.81 & 4.684 \\
7644 & 101947 & 150.52 & 0.249 \\
12166 & 161405 & 150.90 & 0.388 \\
15963 & 207662 & 150.31 & \\
\hline
\end{tabular}

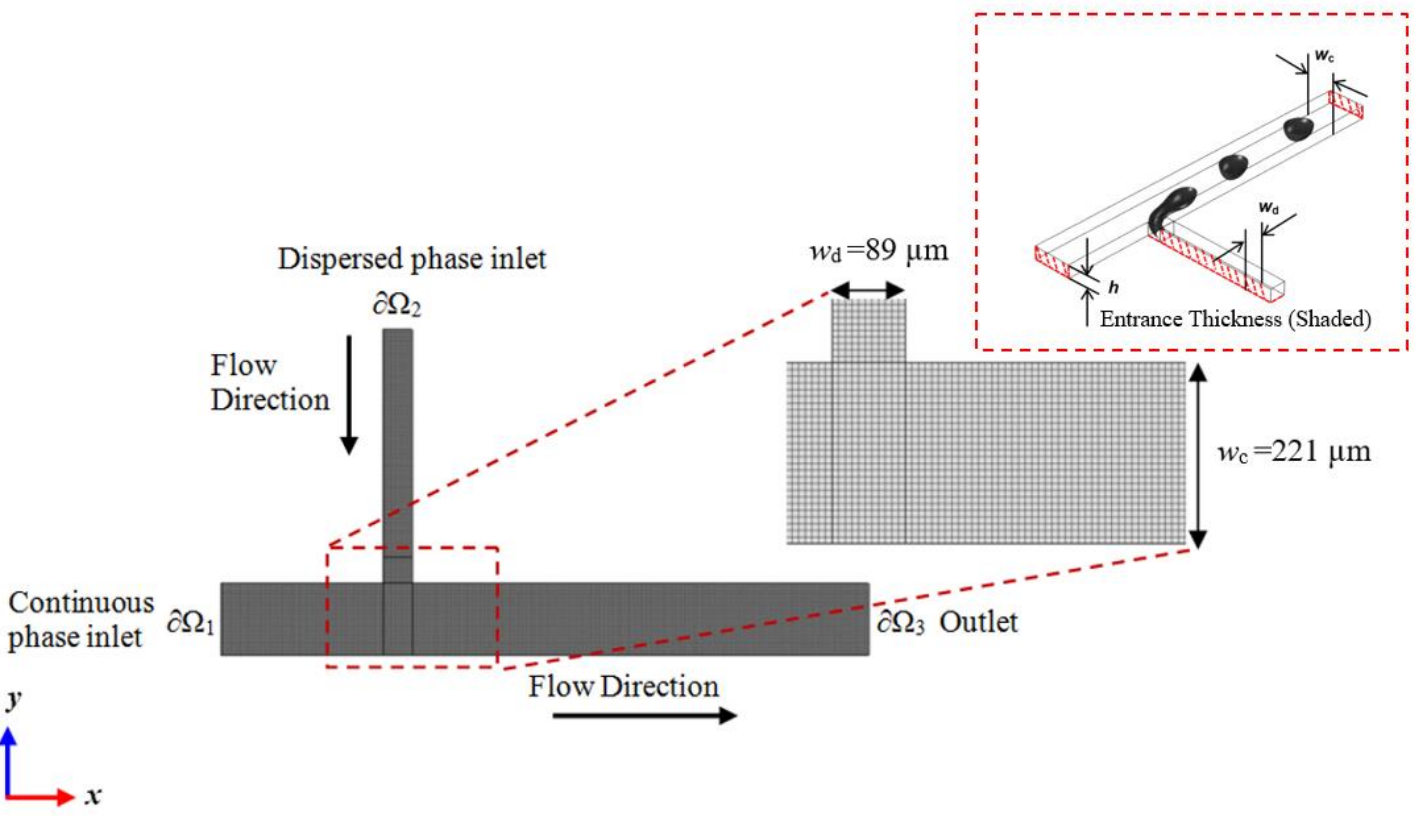

Figure 2: The T-shaped geometry used for the simulations with two-dimensional (2D) quadrilaterals mesh elements.

\section{Mesh Convergence Analysis}

Prior to the parametric study, mesh sensitivity analysis was performed to quantify the 81 dependency of simulation results on mesh size. A flow rate ratio, $Q$ of 0.05 (where $Q_{c}=2.0 \mathrm{ml} / \mathrm{h}$ and $Q_{d}=0.1 \mathrm{ml} / \mathrm{h}$ ) was used in this analysis. The data is recorded at 0.0005 seconds intervals, which were sufficiently small to capture the breakup 84 phenomenon of the fluid-fluid interfaces. A 2D structured mapped mesh was generated and meshes of varying degrees of resolution were set up for the domain. The effect of mesh size was examined by increasing the number of mesh elements 87 from 976 (coarsest grid) to 15963 (finest grid). Table 2 summarized the mesh 
convergence test for the dependence of effective droplet diameter on the number of mesh elements. An acceptable relative error of $0.38 \%$ between the last two finer meshes was considered. The relative error of the measurement shows the error deviation in relation to the measured values of droplet diameter between the two different mesh resolutions. Therefore, an optimal grid resolution containing 7644 elements with cell sizes equal to $0.0082 \mathrm{~mm}$ was selected for the subsequent study.

\section{Numerical Model Validation}

At a very preliminary stage, a validation of the $2 \mathrm{D}$ numerical model of formation of the water droplets was carried out against a matching laboratory experiment. The geometry and the properties of the fluids used in the numerical simulation were set to the values measured in the lab. Deionised water and olive oil (highly refined, low acidity, Sigma Aldrich) were used in the experiment to generate droplets in a Tjunction microchannel.

The comparison with the experiments is carried out to estimate the differences between the numerical and experimental approaches for a known system. Figure 3 illustrates the comparison of effective droplet diameter as a function of the applied flow rate ratio, $Q$, between numerical and experimental results.

As shown in Figure 3, both the experimental measurement and the simulated prediction indicate that the effective diameter of the dispersed phase droplets increases with the increasing flow rate ratio. As the flow rate ratio increases the numerical computations over predicts the effective droplet diameter. The discrepancy between the simulated and the measured droplet diameters is roughly $11 \%$ at the highest flow rate ratio tested and for lower flow rates it shows a reasonably good agreement. Syringe pump induced oscillation of flow rate implied a source of fluctuation in the microfluidic flow, which might affect the droplet diameter. Additionally, the temperature dependence of the physical and rheological properties of each working fluid in experiments may have contributed to the deviation in droplet diameter from numerical simulation as they were assumed to remain constant in the numerical model. Besides, the difficulties of numerical dissipation in advection step of fluid simulation may arise for larger flow rate ratio. In order to reduce the numerical 
dissipation of the scheme, a reasonable range of low flow rate ratio, $0.04 \leq Q<$ 0.0675 , has been selected for the subsequent modelling studies of non-Newtonian flow in order to ensure higher reliability and accuracy of the numerical model.

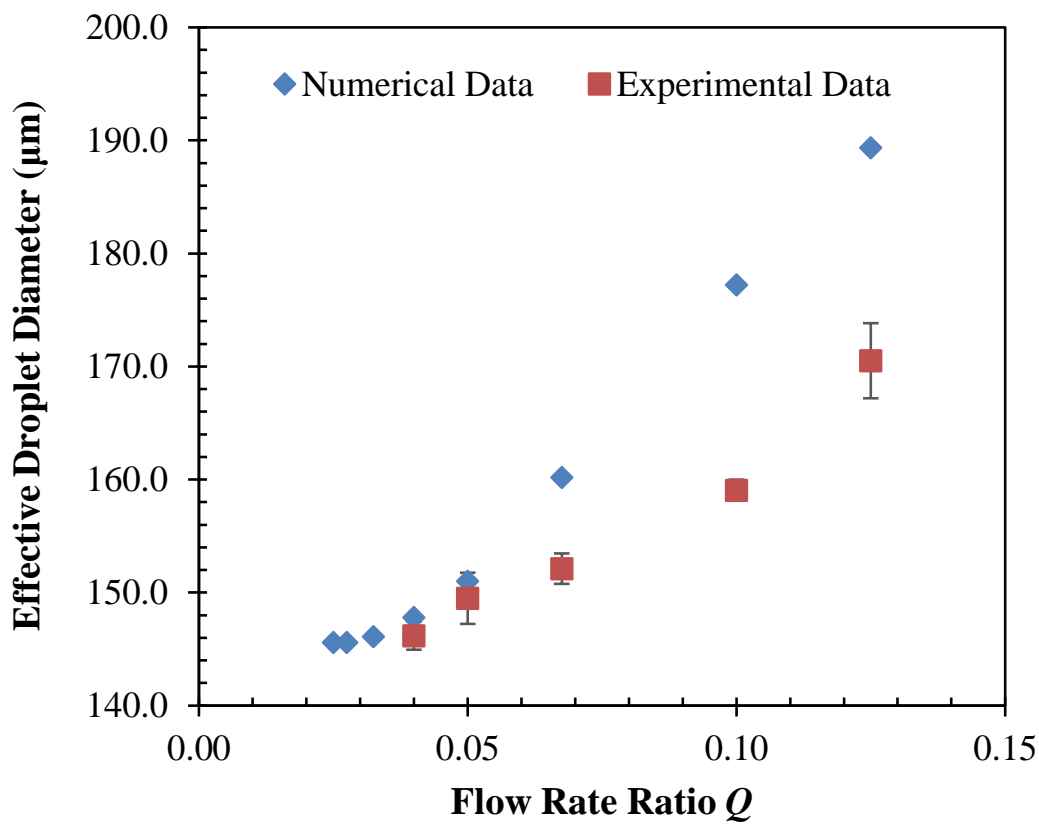

Figure 3: Comparison of effective droplet diameter between 2D numerical and experimental result in the range of $Q$ applied. Error bars indicate the standard deviation in effective droplet size measurement of 30 droplets under fixed experimental condition.

A preliminary validation test of the 3D structure was also performed at the prescribed $Q$ of 0.05 . A grid resolution containing 88257 elements with cell sizes equal to 0.0188 $\mathrm{mm}$ was selected for the study. Figure 4 illustrates a sequence of snapshot of droplet formation for the $2 \mathrm{D}$ and $3 \mathrm{D}$ model representation against laboratory experiments at $Q$ of 0.05 . As seen in Figure 4, a dripping phenomenon, in which droplets are being sheared off before they fill the channel cross-section, were observed for the experimental model. Both $2 \mathrm{D}$ and $3 \mathrm{D}$ simulations predict similar droplet breakup process.

An effective droplet diameter was calculated based on the area of droplet in 2D model, whereas the droplet volume was used to calculate the effective diameter in $3 \mathrm{D}$ model. Note that this approach produces two different values for any given droplet 
as one is on the projected area as seen from the top projection (looking down on to $\left.w_{c}\right)$ and the other is on the volume dispensed based on the total volume fed and the frequency of the droplets. An error of $1.56 \%$ between experimental value of 102.36 $\mu \mathrm{m}$ and numerical value of $100.77 \mu \mathrm{m}$ were obtained in the $3 \mathrm{D}$ case. While the $2 \mathrm{D}$ model presented an error percentage of $2.21 \%$ between the experimental and numerical results of $149.5 \mu \mathrm{m}$ and $152.88 \mu \mathrm{m}$, respectively (see Figure 3). Such discrepancy between 2D and 3D implementation are may attributed to the boundary effects in the third coordinate's direction.

The velocity profiles are not significantly affected in the simplest 2D model when compared to that of 3D channel. A comparison on both, the main and the side channels show (see Figure 5) the maximum velocity remains same while the distribution changed due to wall effects in the 3D case. Nevertheless, based on this comparison, we have concluded that the flow system can be modelled using $2 \mathrm{D}$ geometry with acceptable results compared to $3 D$ and experiments. As it is computationally too costly for extensive tests of 3D model to resolve the typical nanosized interface and motion (Liu and Zhang, 2009) of microdroplets, the present parametric analyses were implemented based on 2D model.

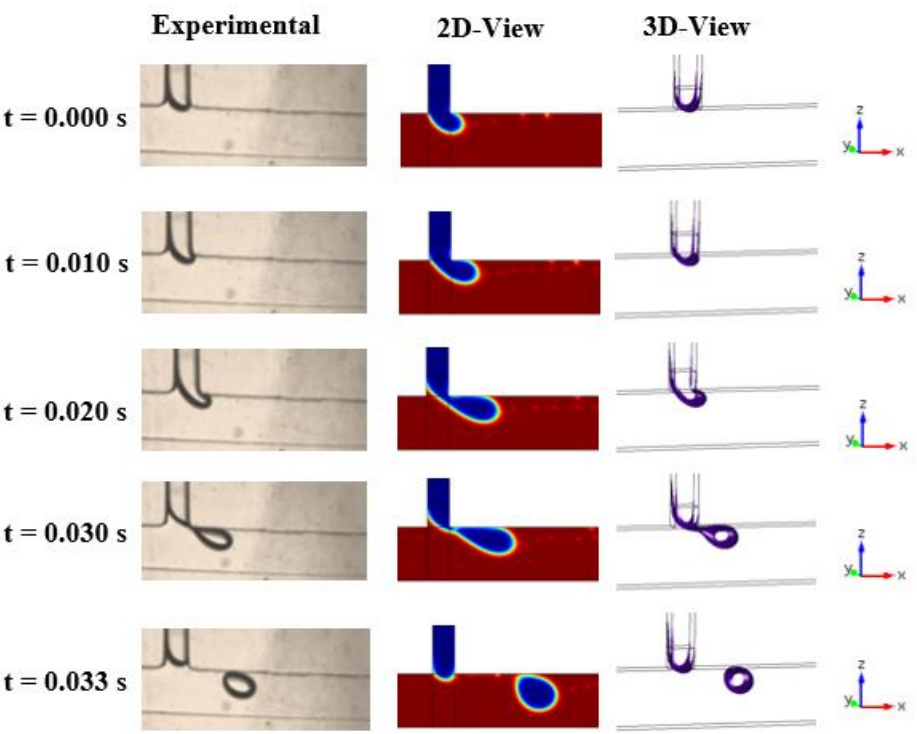

Figure 4: Snapshots of three-dimensional (3D) and two-dimensional (2D) simulations of the water-in-oil droplet breakup process in microchannel and corresponding to experiments (for system: $Q_{\mathrm{c}}: 2.0 \mathrm{ml} / \mathrm{hr}$ and $Q_{\mathrm{d}}: 0.1 \mathrm{ml} / \mathrm{hr}, Q=0.05$ ). The colour bar specifies the dispersed phase as the area where colour distinction is from 0 to 0.5 and continuous phase the area from 0.5 to 1.0 . The iso-surface of 0.5 denotes the interface where the droplet is identified as solid blue rounded shape. 

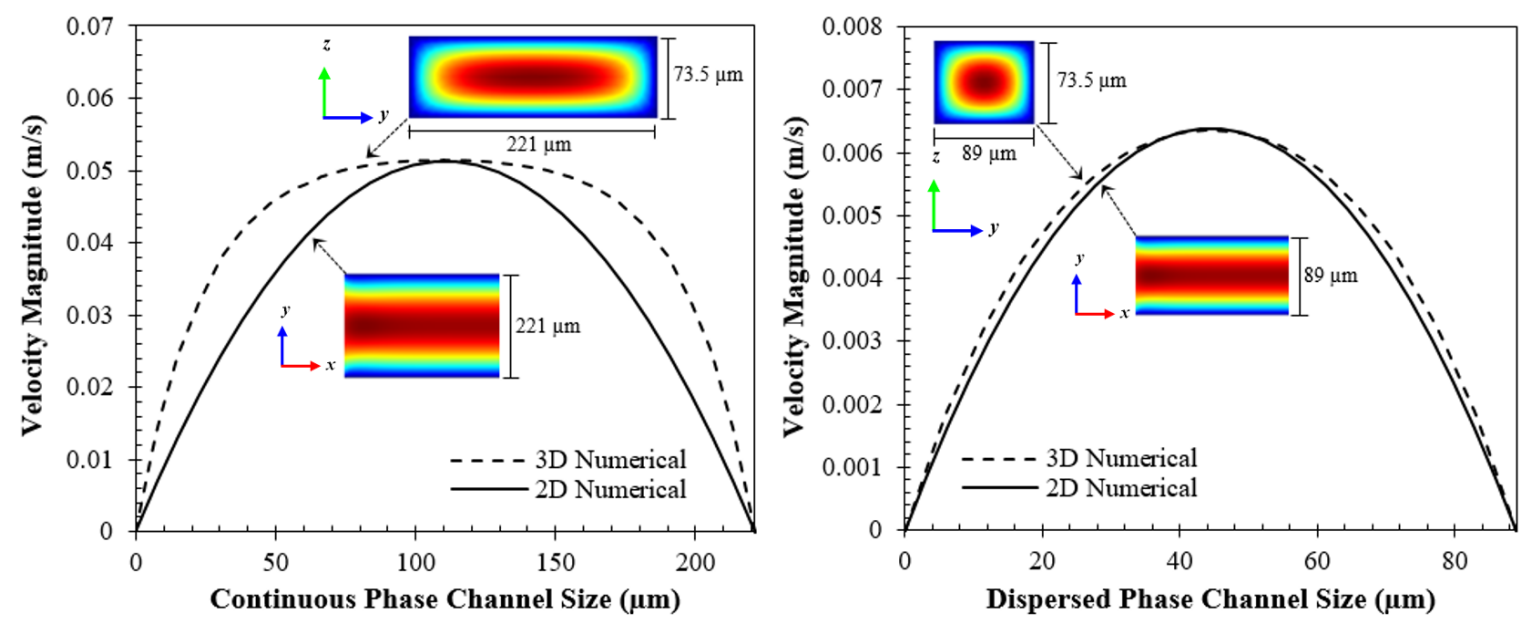

Figure 5: Comparison of velocity profiles for 3D and 2D continuous and dispersed phase microchannel.

\section{Results and Discussion}

\subsection{Droplet formation and breakup mechanism}

The simulations closely follow the experimental observations of the formation and the detachment of a droplet in the microfluidics T-junction. First, the tip of the dispersed phase filament enters the main channel and partially blocks it, which results in the continuous phase having to travel through a smaller cross-sectional area at the Tjunction. This causes higher drag at the interface between the two phases and, over time, the dispersed phase entering the T-junction is slowly convected downstream. Due to the drag applied by the continuous phase, the dispersed phase filament thins out forming a neck. The pressure gradient across the forming liquid filament and the drag at the interface further distort the droplet in the downstream direction until they overcome the surface tension and a droplet is formed (Garstecki et al., 2006; Garstecki et al., 2005; Glawdel et al., 2012). The fully detached droplet flows downstream in the main channel, while the dispersed phase retracts into side channel. This break-up process is continuously repeated, and the size of the droplets produced can be varied by altering the fluid flow rates, the channel dimensions or by changing the physical properties of the two phases. The results in Figure 6 and 7 were computed using the numerical scheme outlined in Section 2 with both the dispersed and continuous phases being Newtonian fluids with viscosities of $9.3 \times 10^{-4}$ Pa.s and $6.8 \times 10^{-2}$ Pa.s, respectively. 


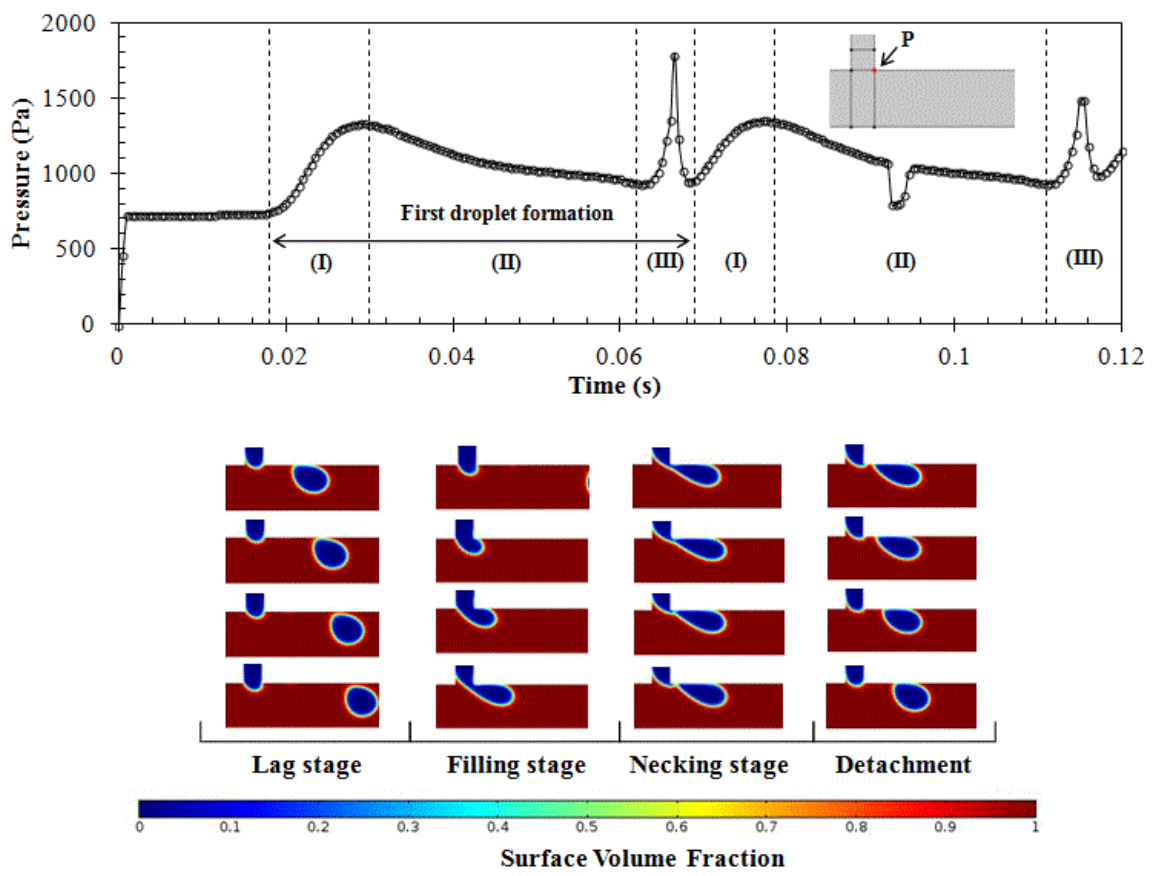

Figure 6: Evolution of the pressure at point $\mathrm{P}$ during droplet breakup. $\mathrm{P}$ denotes the point at the junction and reflects the evolution of breakup mechanism. The droplet formation process can be divided into three stages: (I) lag stage, (II) filling stage, and (III) necking stage.
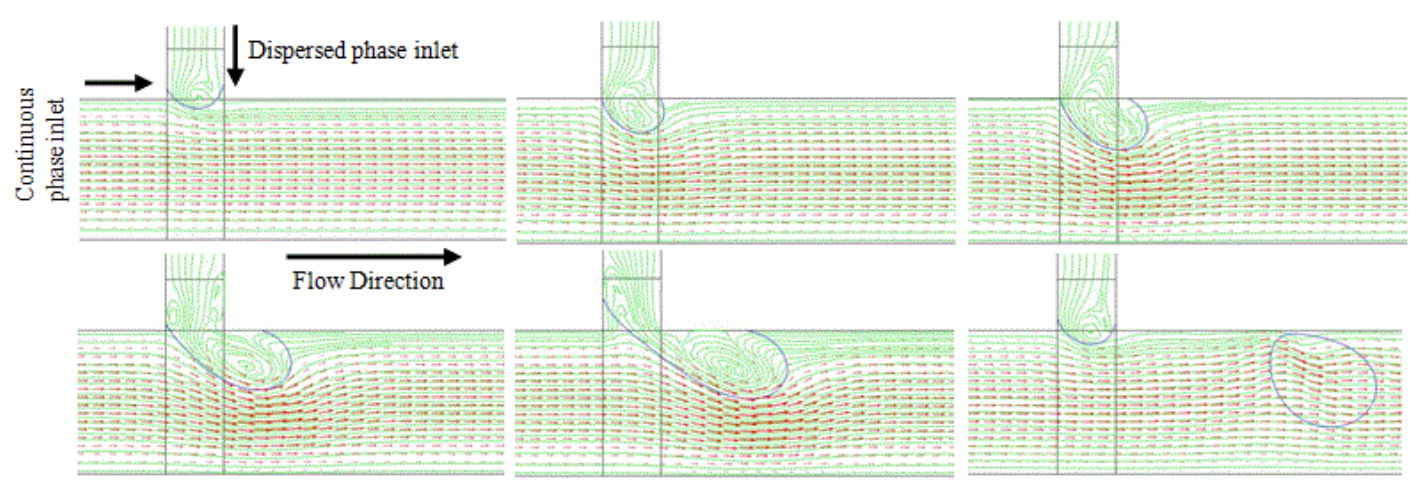

Figure 7: Streamline and velocity field representation of the process of droplet breakup in a model of the T-shaped.

Variations in pressure at the leading edge of the $\mathrm{T}$ junction indicated by point $\mathrm{P}$ during the growth of the dispersed phase filament and the detachment of the drop is given in Figure 6. It shows that the pressure increases gradually until the drop starts to yield under the shear and the pressure build up and then decreases until the breakup. The breakup of the droplet is marked by the sharp peak. Then the filament starts to grow again and the pressure at point $P$ starts to increase. The sudden depression in the pressure during the formation of the second drop indicates first drop exiting the 
computational domain boundary. It should be noted that the pressure inside the droplet is higher than that of the continuous phase in the neighbourhood. Since the boundary condition applied assumes the droplets to be entering the constant pressure as they leave the domain, the pressure field shows a sudden reduction spread over the time it takes a droplet to cross the outlet boundary completely. This phenomenon can be observed in all subsequent parametric studies.

The streamlines are overlaid on the velocity field in Figure 7 to investigate the flow development within the dispersed phase filament and the growing droplet. In Figure 7 , at the start of the formation process, a recirculation flow is generated close to the interface due to the shear applied by the continuous phase flow. This region expands gradually when the dispersed phase enters the main stream and elongates downstream. When detached, the droplet is carried by the continuous phase and the initial flow is disturbed and the recirculation flow no longer exists.

The droplet detachment mechanism has been modelled by Husny and Cooper-White (2006) for Newtonian fluids, and we use this model here to illustrate the influence of physical properties of the fluids on the droplet size. We consider forces and velocities along the main channel. The droplet detaches when it becomes sufficiently large that the cross-flow drag force from the continuous phase, which pulls the droplet downstream, matches the interfacial tension force joining the droplet to the side channel. The interfacial tension force is given by

$F_{\sigma}=\frac{\pi \sigma w_{d}^{2}}{d}$

where $d$ is the droplet diameter. The cross-flow drag force is given by

$F_{D}=3 \pi \eta_{c}\left(v^{*}-v_{d}\right) d f\left(\lambda_{\eta}\right)$

where $v^{*}$ is the continuous phase velocity at the height of the droplet centre, $v_{d}$ is the droplet velocity, $\lambda_{\eta=} \eta_{d} / \eta_{c}$ is the viscosity ratio and $f\left(\lambda_{\eta}\right)=\left(\frac{2}{3}+\lambda_{\eta}\right) /\left(1+\lambda_{\eta}\right)$ captures the effect of the disperse phase viscosity on the droplet drag. When $\lambda_{\eta} \gg 1$ then the disperse phase viscosity is sufficiently high that the droplet is solid-like and equation 17 reduces to the drag from Stokes flow around a solid sphere. Conversely, for smaller values of $\lambda_{\eta}$, internal flow within the droplet becomes possible and this acts to reduce the drag on the droplet from the continuous phase. We note 
that $2 / 3<f\left(\lambda_{\eta}\right)<1$, so this model predicts a fairly modest effect of the disperse phase viscosity.

\subsection{Effect of Dispersed Phase Rate}

In the first series of numerical simulations, the effect of the dispersed phase flow rate, $Q_{d}$, on the droplet diameter was systematically examined for a fluid with physical properties equivalent to SCMC solutions with concentrations ranging from $0.00 \mathrm{wt} \%$ to $1.20 \mathrm{wt} \%$. Similarly, the continuous phase properties were selected to reflect those of olive oil $\left(\eta_{c}=0.068\right.$ Pa.s). A contact angle of $180^{\circ}$, representing complete repulsions of the working liquid by the channel surface was applied. This is to reflect the wall being wetted with the oil, forming a lubricating layer preventing the formation of three phase contact line at the channel surface.

For a constant continuous phase flow rate, $Q_{c}$, of $2.00 \mathrm{ml} / \mathrm{hr}$, the droplet size, $d$, was measured with increasing $Q_{d}$ between $0.05 \mathrm{ml} / \mathrm{hr}$ and $0.135 \mathrm{ml} / \mathrm{hr}$. Results are shown in Figure 8. Here $d$ increases with increasing $Q_{d}$ and with increasing SCMC concentration and, as $Q_{d}$ increases then the results for different SCMC concentration becomes more tightly bunched. The droplet size increases with $Q_{d}$ as, for larger $Q_{d}$, larger volumes of the dispersed phase fluid enter the main channel before the filament is pinched off. The increase in drop size does not replicate the threefold increase of the dispersed phase flow rate, because the formation time shortens. However, it should be noted that the frequency of droplet shedding increases in line with conservation of mass. The effect of SCMC concentration can be explained as follows. Figure $1 \mathrm{~b}$ shows that, for all $Q_{c}$ in this study, there is a considerable increase in the effective $\eta_{d}$ with SCMC concentration even when shear thinning is accounted for. This increase in $\eta_{d}$ leads to smaller droplets because, as equation 17 shows, larger $\eta_{d}$ leads to an increase in the cross-flow drag force, due to internal flow inside the droplet being suppressed. This effect, combined with the shear thinning, also explains why the results at different SCMC concentrations become more closely packed with increasing $Q_{d}$. As $Q_{d}$ increases flow within the droplets becomes faster and hence shear thinning lowers the effective viscosity. As figure 1 shows, shear thinning is stronger in the more concentrated SCMC solutions, thus with increasing flow rates the range of effective viscosity ratios narrows and so does the range of droplet sizes. 


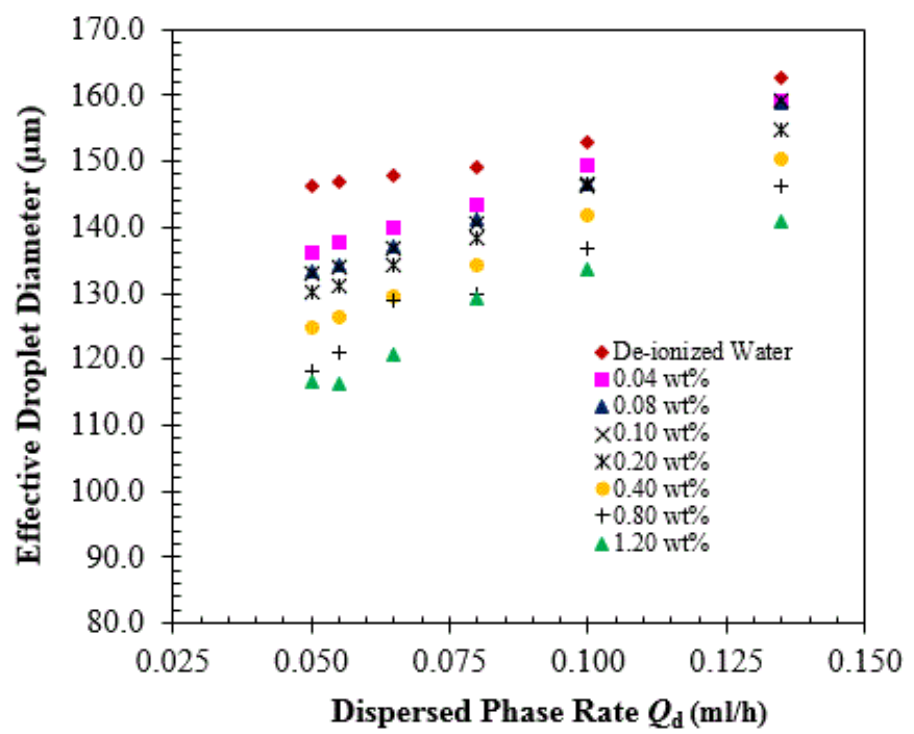

Figure 8: Effect of $Q_{d}$ on the effective droplet diameter of SCMC solutions for various SCMC concentrations. The continuous phase flow rate $Q_{c}$ is kept constant at $2.00 \mathrm{ml} / \mathrm{hr}$ for all cases reported.

The time dependence of the pressure at the corner of T-junction during SCMC droplet breakup at different $Q_{d}$ were systematically investigated and are plotted in Figure 9. At the necking point, there is a pressure spike at the time of detachment. Moreover, the magnitude of pressure depends on the specific location of the droplet detachment. When the $Q_{d}$ is small, the droplets are pinched off at the T-junction. When $Q_{c}>Q_{d}$, droplet does not have time to fill the main channel fully producing elongated slugs. The droplet breakup occurs immediately after a short filament is formed filling the main channel partially and the droplet detachment occurs at the junction ${ }^{41}$. These observations are also supported by Tan et al. (2008) who reported that the shear force from the continuous phase is sufficiently large to induce the dispersed phase to form droplets at the T-junction of the microchannel at the low dispersed phase rates. When $Q_{d}$ increases, the filament grows at faster rates, partially blocking the main channel. This leads to detachment of the droplets at increasing frequency. This is clearly shown in the Figure 9. The distinct pressure peak at point $P$ at the corner decreases as the filament no longer retracts into the side channel. As the $Q_{d}$ increases further, the filament begins to extend in the main channel and the droplet detachment takes place downstream of the T-junction. As the filament grows beyond the corner of the Tjunction, the pressure at corner of the junction becomes less important. Downstream 
detachment of the droplets occurs more readily for fluids with high $\eta_{d}$ because they are more able to resist fragmentation of the filament of disperse phase as it forms.
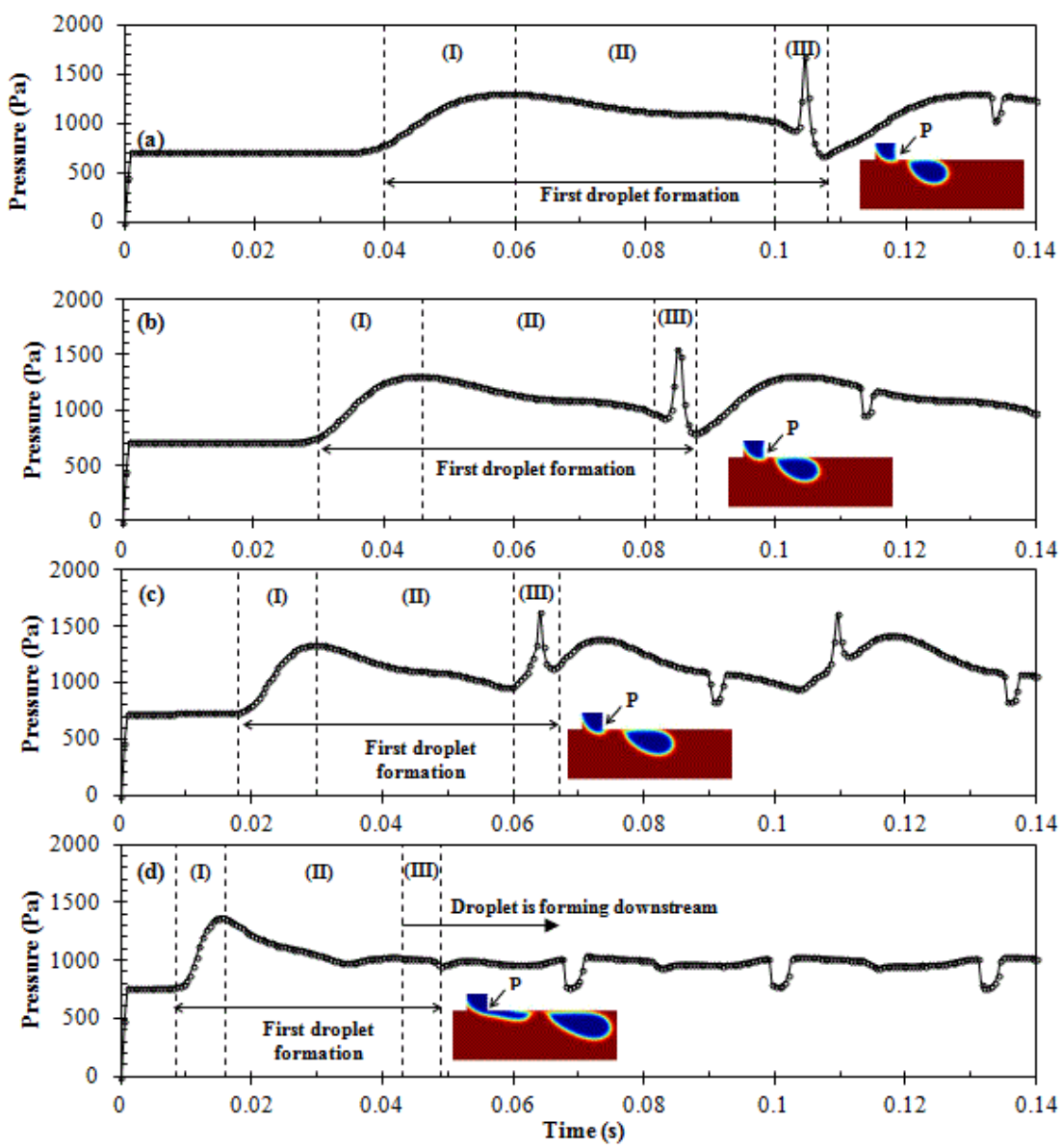

Figure 9: Pressure profile of $0.20 \mathrm{wt} \% \mathrm{SCMC}$ for the effects of $Q_{d}$ on SCMC droplet breakup process at each $Q_{d}$ : (a) $0.050 \mathrm{ml} / \mathrm{hr}$, (b) $0.065 \mathrm{ml} / \mathrm{hr}$, (c) $0.100 \mathrm{ml} / \mathrm{hr}$, (d) $0.200 \mathrm{ml} / \mathrm{hr}$ (for system: $Q_{\mathrm{c}}=2.00 \mathrm{ml} / \mathrm{hr}$ ). P denotes the pressure at the corner of Tjunction. The sharp peak with given data values reflects the droplet breakup at the corner of the side channel as shown in the extracted images. At high $Q_{d}$, the pressure peak becomes insignificant as the detachment point is located downstream of the junction.

\subsection{Effect of Continuous Phase Rate}

As discussed in section 5.1, droplet formation at the T junction is dominated by the balance of the interfacial force with the viscous stresses imposed by the continuous phase. The size of the droplets can be tuned by changing $Q_{c}$, which alters the viscous stresses on the droplets (DeMenech et al., 2008). Since the channel width of the continuous phase $\left(w_{c}=221 \mu \mathrm{m}\right)$ is larger than dispersed phase channel width $\left(w_{\mathrm{d}}=\right.$ $90 \mu \mathrm{m}$ ), the dispersed phase stream does not occupy the entire width of the channel 
downstream of T-junction. Thus the breakup is controlled by the local shear-stress of the continuous phase acting on the dispersed phase droplet, which can be estimated via equation 17. For a constant $Q_{d}$ at $0.20 \mathrm{ml} / \mathrm{h}$, the droplet diameter was measured with the variation in $Q_{c}(2.50 \mathrm{ml} / \mathrm{h}$ to $5.00 \mathrm{ml} / \mathrm{h})$. Results are illustrated graphically in Figure 10.

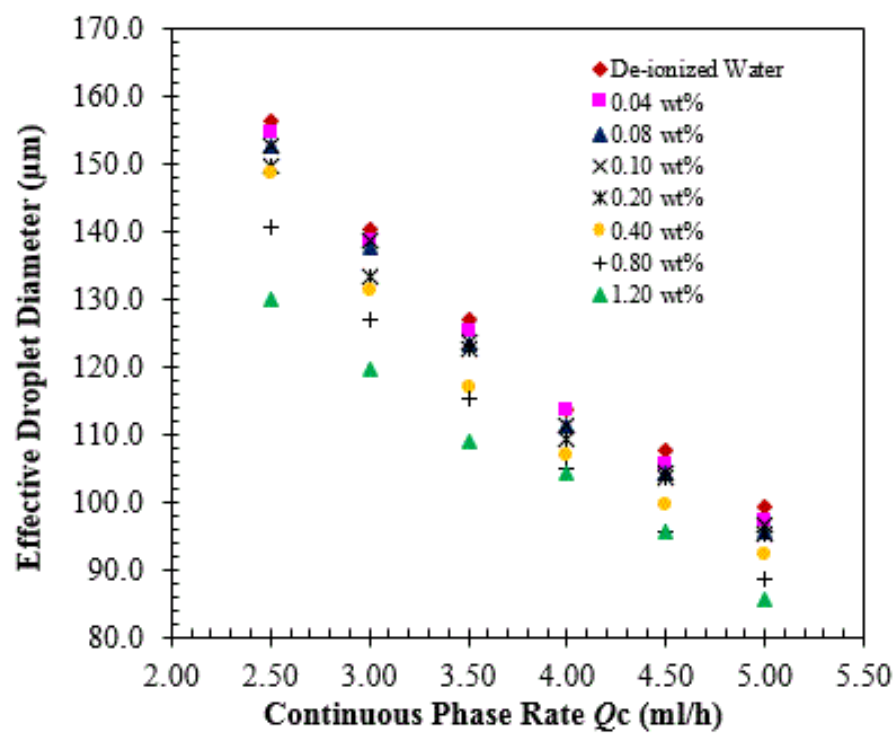

Figure 10: Effect of $Q_{c}$ on the effective droplet diameter of SCMC solutions for various SCMC concentrations. The dispersed phase flow rate $Q_{d}$ is kept constant at $0.20 \mathrm{ml} / \mathrm{h}$ for all cases reported.

Figure 10 shows that SCMC droplet size can be controlled in the T-junction microchannel by varying the flow rates of the continuous phase when the disperse phase is kept constant. As $Q_{c}$ is increased, the simulation results show a reduction in droplet size (see Figure 10). This is because at higher $Q_{c}$, the shearing effect imparted by the continuous phase on the dispersed phase is larger, and as a result, smaller droplets will be pinched off. According to Husny et al. (2003), this is because, a higher $Q_{c}$ imparts a higher cross-flow drag force on the droplet. Consequently, this leads to a decrease in the droplet growth time, resulting in a reduction in final droplet volume. This result also agrees with Thorsten et al. (2001) and Tan et al. (2008) who reported that droplet size decreased with increased continuous phase flow rate. Figure 10 also shows that decreased SCMC concentration leads to larger droplets. This is for the 
same reason as in Figure 8, namely that a lower disperse phases viscosity enables stronger internal flow in the droplet, reducing the cross-flow drag force. Also similarly to Figure 8 , increasing the flow rate $Q_{c}$ causes the variation of droplet size with SCMC concentration to lower. As above, this is because the more concentrated SCMC solutions have a larger zero shear viscosities but also stronger shear thinning. Hence increasing $Q_{c}$ narrows the separation of $\eta_{d}$ values across the concentration range (see figure 1b). With increased SCMC concentration we also see increased formation of extended filaments into the downstream region. The increased $\eta_{d}$ opposes the breakup of the disperse phase filament and results in longer threads (Zhang and Basaran, 1995; Nunes et al., 2013). This prediction is corroborated by reported experimental observations at a T-junction microfluidics (Gu and Liow, 2011).

Figure 11 illustrates the evolution of pressure of the entire droplet formation process as observed at the trailing edge of the side channel (marked by point $P$ in the subfigure) for $0.20 \mathrm{wt} \% \mathrm{SCMC}$ at each continuous phase flow rate. The average pressure increases from Figure 11 (a) to (d) in accordance with the increase of the flow rate. However, the pronounced sharp pressure peaks, as previously mentioned in section 5.1, are not observed at lower $Q_{c}$. As $Q_{c}$ decreases, the position of the necking and detachment point is shifted downstream, leading to the jetting phenomenon where a long neck of dispersed thread is developed before the droplets are sheared off in the main channel. It is worth noting that the pressure at which the droplets pinch off initially varies slightly, as it takes several cycles of droplet production to reach steady state. As $Q_{c}$ increases, the gap between the emerging droplet and main channel wall is increased. Here the emerging dispersed phase filament does not have sufficient time to obstruct the main channel before detachment. 


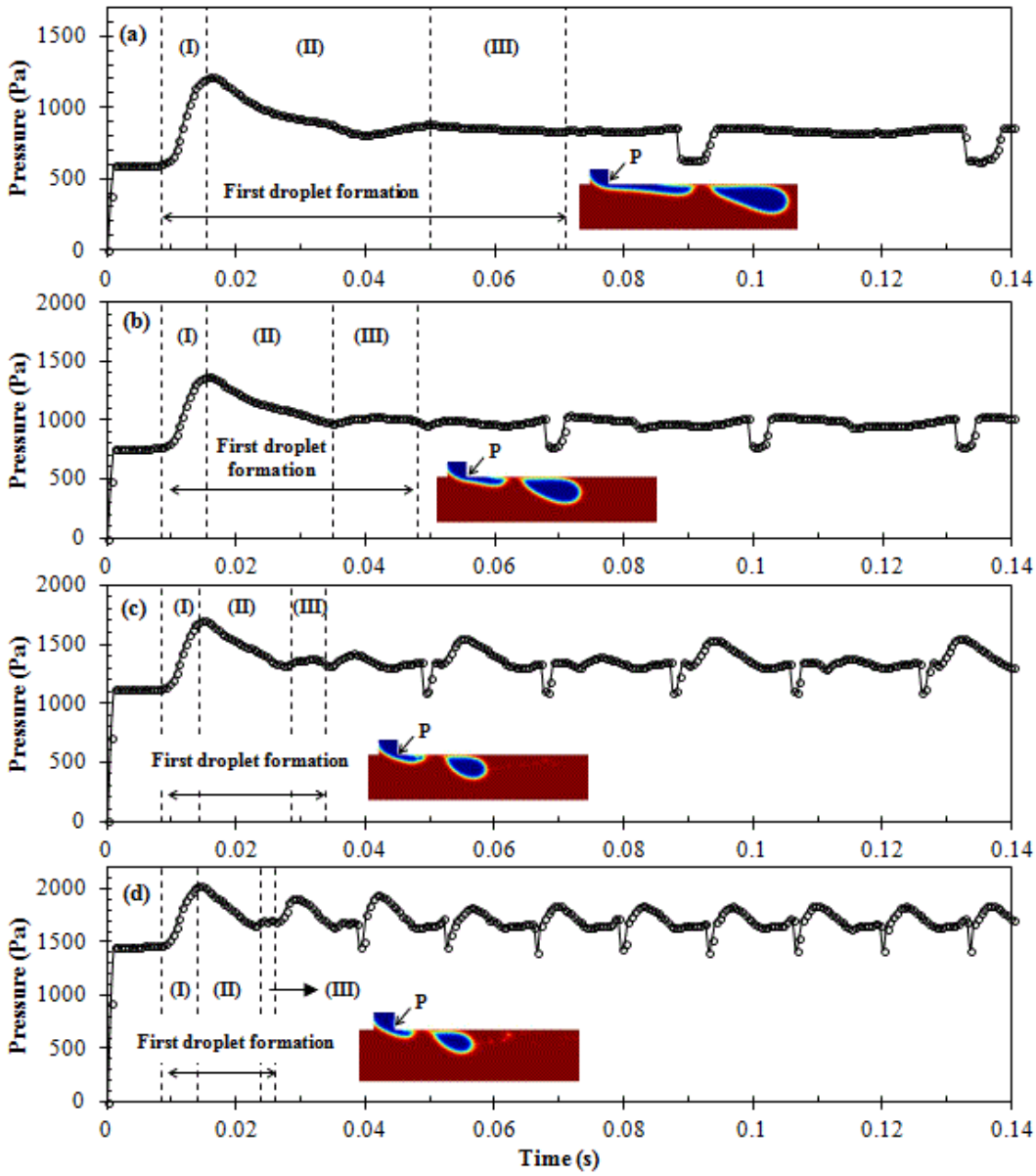

Figure 11: Pressure profile of 0.20 wt $\%$ SCMC for the effects of $Q_{c}$ on SCMC droplet breakup process at each Qc: (a) $1.50 \mathrm{ml} / \mathrm{hr}$, (b) $2.00 \mathrm{ml} / \mathrm{hr}$, (c) $3.00 \mathrm{ml} / \mathrm{hr}$, (d) 4.00 $\mathrm{ml} / \mathrm{hr}$ (for dispersed phase flow rate $Q_{\mathrm{d}}=0.20 \mathrm{ml} / \mathrm{h}$ ). $P$ denotes the pressure at the corner of T-junction. After the first droplet is pinched off, the pressure fluctuates abruptly and repeatedly. The lack of a sharp pressure peak occurs when the detachment point is located downstream of the junction. 


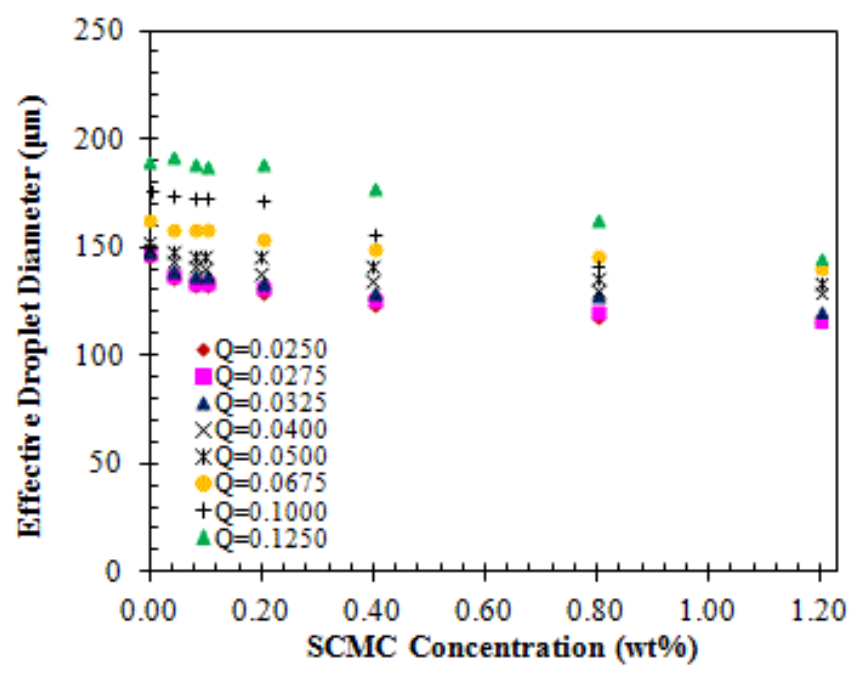

Figure 12: Effect of SCMC concentration on effective droplet diameter for various flow rate ratios $Q$ (with fixed $Q_{c}=2.00 \mathrm{ml} / \mathrm{h}$ and $0.05 \mathrm{ml} / \mathrm{h}<Q_{d}<0.25 \mathrm{ml} / \mathrm{h}$ ).

\subsection{Effect of Sodium Carboxymethylcellulose (SCMC) Concentration}

The present parametric analysis aims to assess the role of the dispersed phase SCMC concentration on the formation of droplets in a Newtonian continuum. The viscosity and degree of shear thinning both increase with the increasing concentration and molecular weight. Figure 12 plots our results to emphasize the influence of SCMC concentration. Increasing the concentration of SCMC reduces $d$, and increasing the flow rate reduces the influence of concentration. These two effects are explained by the viscosity and degree of shear thinning, respectively, and their increase with concentration (see Figure $1 \mathrm{~b}$ and equation 17). This is consistent with experiments by $\mathrm{Gu}$ and Liow (2011) who saw a decrease of droplet size with increasing polymer concentration, for fluids in which the polymers increase the viscosity and the strength of shear thinning. There is a notable contrast with experiments of Husny and CooperWhite (2006) who reported no observable change in droplet diameter with increasing polymer molecular weight but a strong change in the filament characteristics. However, their polymer solutions were Boger fluids, meaning that the polymer influenced the elasticity but not the shear viscosity. This illustrates the potential to tailor the droplet size and formation mechanism by controlling the shear-thinning and elasticity through choice of polymer concentration and molecular weight. 
592 Figure 13 demonstrates the pressure distribution during droplet breakup at the T593 junction corner for the concentration of $0.00 \mathrm{wt} \%, 0.20 \mathrm{wt} \%$ and $0.80 \mathrm{wt} \%$ SCMC

601

612

\subsection{Effect of Continuous Phase Viscosity}

Several types of oil with different viscosities, namely mineral oil, olive oil and peanut oil, were modelled as the continuous phase (From data in Loizou et al. (2014)). Table 3 shows the experimental data of viscosities and interfacial tensions of different continuous phase systems and we note that as $\eta_{c}$ increases the interfacial tension decreases, both of which are associated with smaller droplets. Numerical simulations were performed to examine the effect of the continuous phase on the SCMC droplet breakup process at a constant $Q$ value of 0.05 (where $Q_{d}=0.10 \mathrm{ml} / \mathrm{hr} ; Q_{c}=2.00$ $\mathrm{ml} / \mathrm{hr})$. 

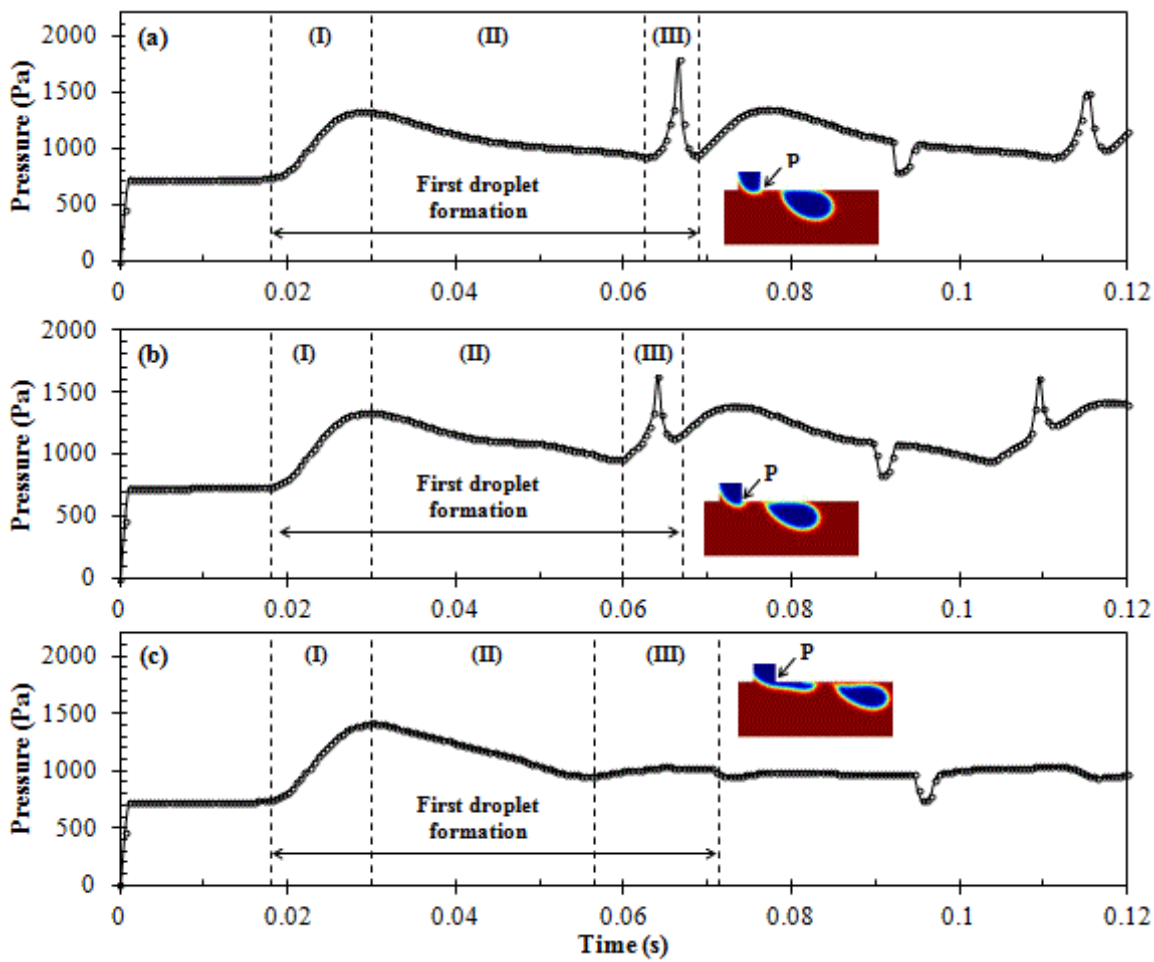

Figure 13: Pressure profile of (a) $0.00 w t \%$, (b) $0.20 w t \%$, (c) $0.80 w t \%$ SCMC droplet breakup process (for system: $Q_{d}=0.10 \mathrm{ml} / \mathrm{h} ; Q_{c}=2.00 \mathrm{ml} / \mathrm{h}$ ). P denotes the pressure at the corner of T-junction. The sharp pressure peak reflects the droplet breakup point as shown in the extracted images. The absence of a sharp pressure peak means that the detachment point is located downstream of the junction.

These results are presented in Figure 14, which illustrates that the droplet diameter decreases as $\eta_{\mathrm{c}}$ increases, as anticipated. Larger $\eta_{\mathrm{c}}$ produces a larger drag force from the continuous phase onto the droplet, meaning that it detaches at a smaller size (see equation 17). Data from Husny and Cooper-White (2006) show that increasing the viscosity of continuous phase decreases the droplets size. The findings of this study are also consistent with those earlier experimental studies (Garstecki et al., 2006; Gu and Liow, 2011; Yeom and Lee, 2011). For the oils we modelled, the interfacial tension also reduces as the viscosity increases and this is expected to further reduce the droplet size, as the interfacial tension resists detachment of the growing droplet (see equation 16). Indeed, Figure 14 shows that the properties of the continuous phase strongly influence the droplet size, whereas the disperse phase properties, which are varied via the SCMC concentration, have a smaller secondary effect. 
640 Figure 15 illustrates the variation in SCMC droplet diameter for different viscosities of the continuous phase. As above, increased viscosity ratio reduces the droplet size, but this effect is small compared to the influence of the continuous phase viscosity. We note that the strongest effect of the SCMC occurs at lower concentrations where the difference in viscosity ratio between neighbouring concentrations is most pronounced (see Figure 1b). The pressure profile of $0.20 \mathrm{wt} \%$ SCMC droplets under different $\eta_{\mathrm{c}}$ at fixed flow rate ratio $Q$ are illustrated in Figure 16. As observed from Figure 16, the build-up of the pressure at the corner of the junction increases as $\eta_{c}$ decreases. The pressure peaks are less marked in the case of larger $\eta_{\mathrm{c}}$. This appears to correlate with droplet size and also downstream distance of the detachment point. Consequently, sharp peaks were clearly observed only for smaller $\eta_{\mathrm{c}}$.

\begin{tabular}{lll}
\hline $\begin{array}{l}\text { Continuous } \\
\text { Phase System }\end{array}$ & Dynamic Viscosity (mPa.s) & $\begin{array}{l}\text { Interfacial tension between oil } \\
\text { and water (Owt\% SCMC) } \\
(\mathrm{mN} / \mathrm{m})\end{array}$ \\
\hline Mineral oil & $35.40 \pm 0.00$ & $41.37 \pm 2.90$ \\
Olive oil & $68.10 \pm 0.67$ & $20.74 \pm 0.47$ \\
Peanut oil & $98.80 \pm 0.39$ & $18.80 \pm 1.31$ \\
\hline
\end{tabular}

652

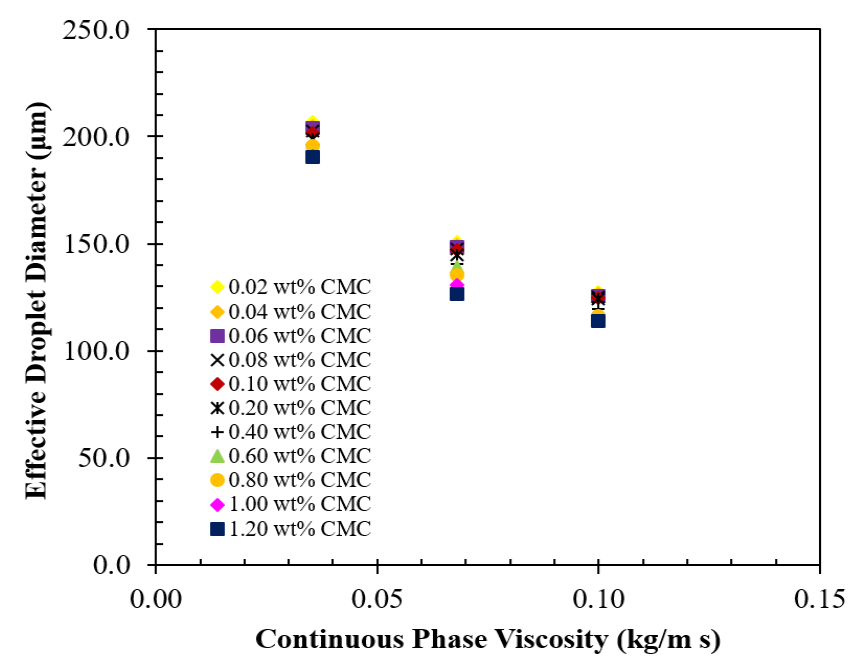

Figure 14: Effect of $\eta_{\mathrm{c}}$ on effective droplet diameter of SCMC droplets (for system: $Q_{d}=0.10 \mathrm{ml} / \mathrm{hr} ; Q_{c}=2.00 \mathrm{ml} / \mathrm{hr}$ ). 


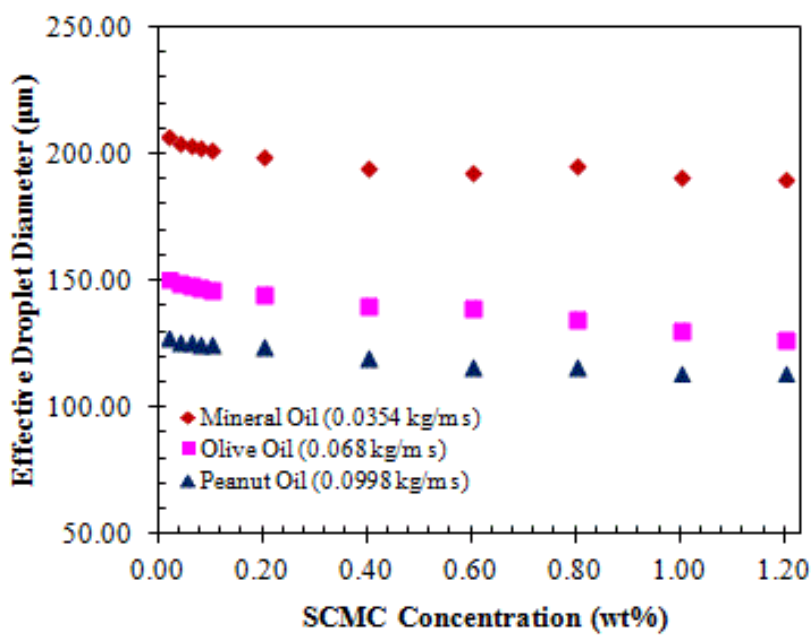

Figure 15: Effect of $\eta_{\mathrm{c}}$ on effective droplet diameter of SCMC droplets (for system:
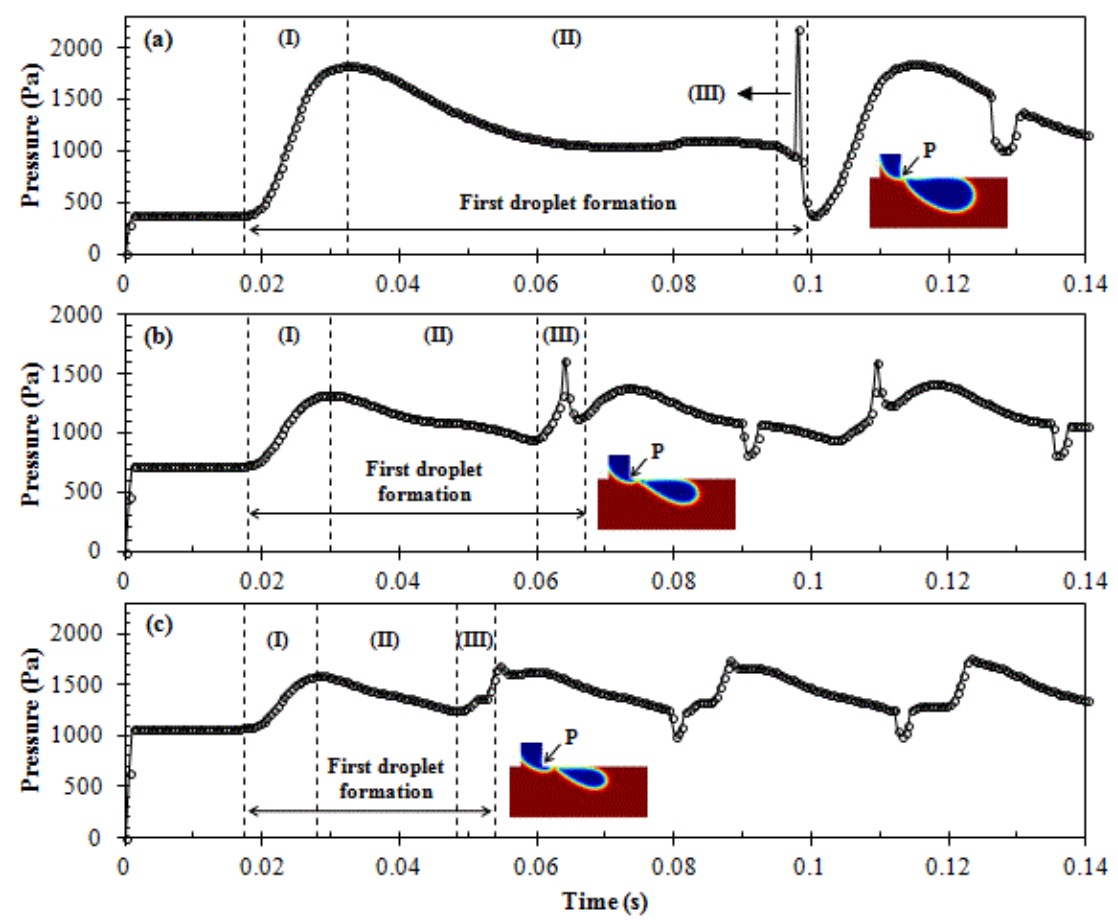

Figure 16: Pressure profile of $0.20 \mathrm{wt} \%$ SCMC droplets breakup for the system with different continuous phase viscosities: (a) Mineral oil $\left(\eta_{c}=0.0354\right.$ Pa.s), (b) Olive oil $\left(\eta_{\mathrm{c}}=0.068\right.$ Pa.s), (c) Peanut oil $\left(\eta_{\mathrm{c}}=0.0998\right.$ Pa.s) (for system: $Q_{\mathrm{d}}=0.10 \mathrm{ml} / \mathrm{hr} ; Q_{\mathrm{c}}=$ $2.00 \mathrm{ml} / \mathrm{hr}$ ). $\mathrm{P}$ denotes the pressure at the corner of T-junction. The sharp peak with given data values reflects the droplet breakup point as shown in the extracted images. The pressure peak becomes insignificant when the detachment point is 


\subsection{Effect of Contact Angle}

673 The influence of the contact angle $(\theta)$ on drop shape size and production has been

674 the focus of several investigations of Newtonian fluids (Bashir et al., 2011; 675 Kumacheva and Garstecki, 2011). However the role of the contact angle on nonNewtonian droplets with shear-thinning behaviour has not been widely studied. Due to the resulting high surface area to volume ratios of micro-sized devices, the interaction between the solid surface of the microchannel and the fluid has been a major focal point in affecting the dynamics of the droplet formation process. In a microfluidic geometry with a T-junction configuration, the continuous and dispersed phases were initially dispensed separately at the desired flow rates through the microchannel with hydrophobic walls. The continuous phase needs to wet the channel walls preferentially in order to separate the dispersed phase, which tends to be non-wetting, from the walls and eventually it breaks into droplets. The propensity of the two flowing immiscible liquids to wet the channel walls is of utmost importance for the droplet formation process to take place and be stable (Rosengarten et al., 2006). The magnitudes of surface wettability can be characterized by static contact angles, whereas the dynamic effects are not explicitly taken into account.

A focus of the present study is to highlight the effect of the wetting characteristic of the channel walls on the non-Newtonian SCMC droplet formation process. The contact angle of the SCMC droplets at the channel walls was varied from $130^{\circ}$ to $180^{\circ}$ and implemented in the simulations. Considering a three-phase coexisting system, this range of contact angles covers partial wetting behaviour to complete non-wetting behaviour. Olive oil was selected as the carrier fluid along the main channel, while a SCMC polymer solution was used as the dispersed phase fluid supplied from the orthogonal channel. Figure 17 illustrates the effect of different wetting conditions on the droplet diameter of each SCMC concentrations. As elucidated in Figure 17, the droplet diameter decreases as $\theta$ increases for a fixed flow rate ratio $Q$ of 0.05 , for the lower $\mathrm{CMC}$ concentrations. Here, $\theta$ has a strong impact on the reduction in droplet size for concentrations from $0.02 \mathrm{wt} \%$ to $0.80 \mathrm{wt} \%$, causing a step change in droplet diameter at about $150^{\circ}$. Interestingly, the effect of $\theta$ on the droplet diameter was found to be opposite at higher concentration at $\mathrm{CMC}$ solution (>0.80 wt\%), showing a weak increase with increasing $\theta$ for almost all rates. 
704 In addition, the numerical simulation predicts that the droplet diameter was observed to be largely independent of $\theta$ when it is larger than $150^{\circ}$, which is in agreement with numerical results from Sang et al. (2009) and Bashir et al. (2011). Sang et al. (2009) reported that larger contact angles $\left(\theta \geq 165^{\circ}\right)$, corresponding to poor wettability of the dispersed phase to the channels wall, had a negligible effect on the resulting diameter of 1,6-hexanediol diacrylate droplet in polyvinyl aqueous solution, in their VOF simulations. Bashir et al. (2011) also reported that the size of the resulting water droplets in $\mathrm{n}$-Dodecane oil solution remains constant in the super-hydrophobic $(\theta$ $\geq 165^{\circ}$ ) regime using LSM. For smaller $\theta$, Liu and Zhang (2009) reported that the droplet interfaces are prone to be normal to the channel walls.

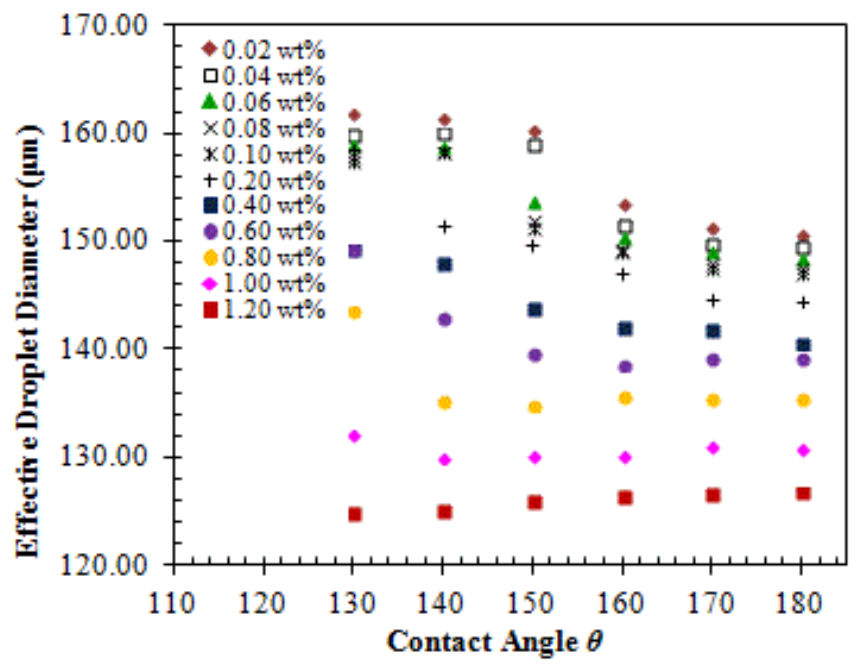

Our simulations show that decreasing the contact angle promotes the formation of downstream filaments. For example, in Figure 18 decreasing $\theta$ causes the formation mechanism to change from corner detachment to downstream detachment via a filament, because the dispersed thread has strong tendency to attach to the wall surface at smaller $\theta$. Thus a larger contact angle reduces the adhesion force between the dispersed fluids with the channels wall, meaning that the cross-flow drag force will detach the droplet earlier, leading to smaller droplets (Bashir et al., 2011; Davidson and Cooper-White, 2006). When the concentration increases above 0.80 
$w t \%$, the droplet diameter increases with increasing $\theta$, but the dependence was very slight (see Figure 17). Correspondingly, Figure 19 shows higher SCMC concentrations and for these high-viscosity disperse phases, the filament is present even at high $\theta$. Thus increasing $\theta$ merely shortens the filament and does not remove it. As there is no change in the mechanism, the effect of $\theta$ is weaker at these higher SCMC concentrations. Indeed for the highest SCMC concentrations the filament is already so long that the corner has virtually no effect. The solutions, in these cases, appear to be self-similar, with $\theta$ determining the downstream position of the detachment but with little effect on the detachment dynamics. Hence, once the initial filament is formed, $\theta$ has very little effect on the droplet size.

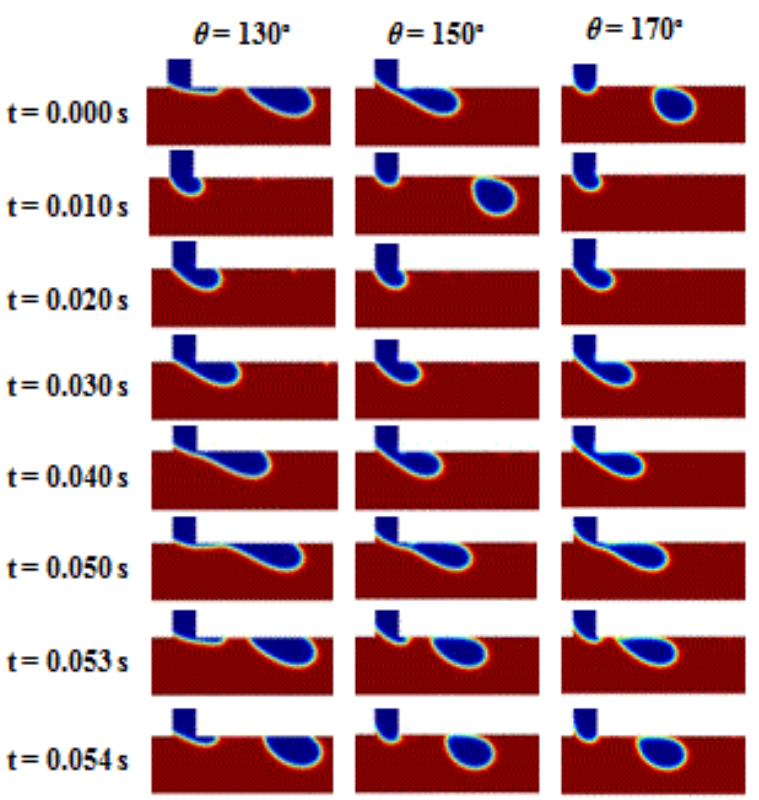

Figure 18: Snapshots of two-dimensional (2D) simulations of the effect of contact angle on 0.20 wt $\%$ SCMC-in-olive oil droplet breakup process at different wetting conditions: (a) $\theta=130^{\circ}$ (b) $150^{\circ}$ (c) $\theta=170^{\circ}$ (for system: $Q_{d}=0.10 \mathrm{ml} / \mathrm{hr} ; Q_{c}=2.00$ $\mathrm{ml} / \mathrm{hr})$.

Our above findings are confirmed in Figure 20, which shows the variation in droplet diameter for various concentrations at fixed $\theta$. Here, the droplet diameter decreases with increased SCMC concentration, for the reasons discussed in section 5.2, and $\theta$ has less influence over the droplet diameter at higher viscosity because the droplets form via a downstream filament for all $\theta$ values. 
(a)

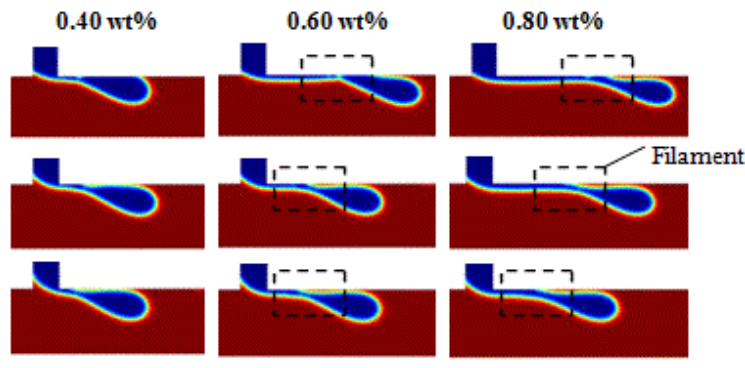

(a)

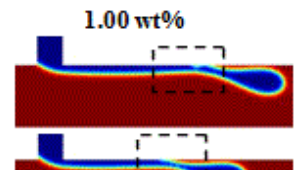

(b)
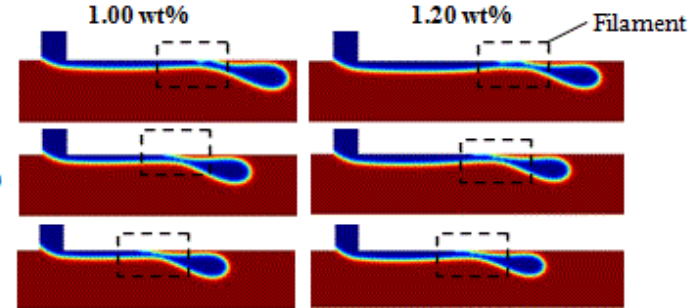

Figure 19: Filament thinning profile prior to pinch-off for SCMC concentrations above $0.40 w t \%$ at contact angle: (a) $130^{\circ}$, (b) $150^{\circ}$, (c) $170^{\circ}$ (for system:

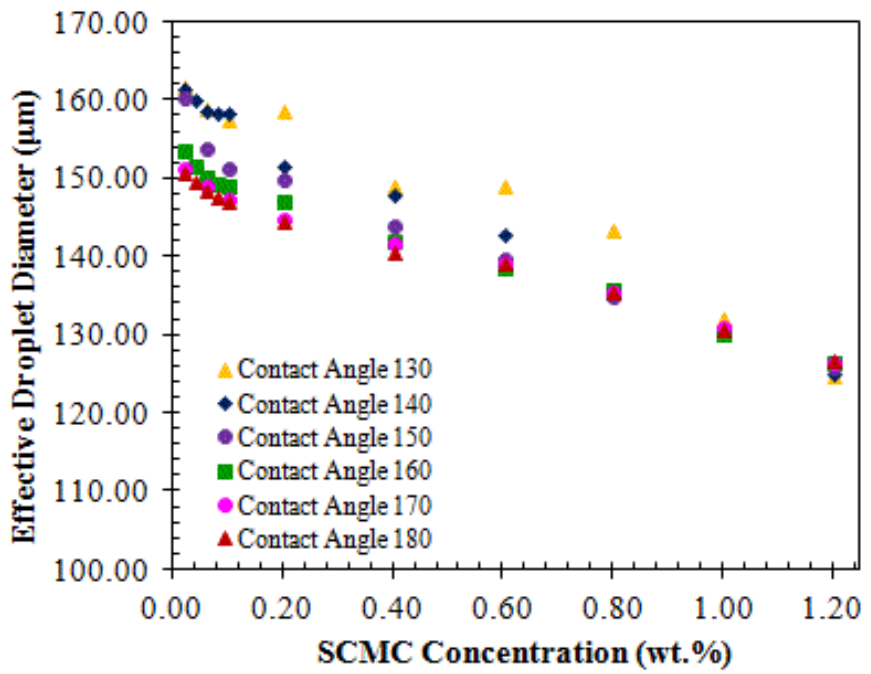

Figure 20: Variation in effective droplet diameter of SCMC droplets for various SCMC concentrations at each wetting condition (for system: $Q_{\mathrm{d}}=0.10 \mathrm{ml} / \mathrm{hr} ; Q_{\mathrm{c}}=$ $2.00 \mathrm{ml} / \mathrm{hr})$. 
Figure 21 illustrates the pressure profile of $0.20 \mathrm{wt} \%$ SCMC droplet breakup at different wetting conditions. On the basis of the results shown in Figure 21, smaller pressure peaks for the breakup process were found for the smaller $\theta$. This is because the wettability drives the fluid toward the surface, thus the contact area between the droplets and solid surface increases which contributes to an elongation of the filament prior to droplet pinch off.
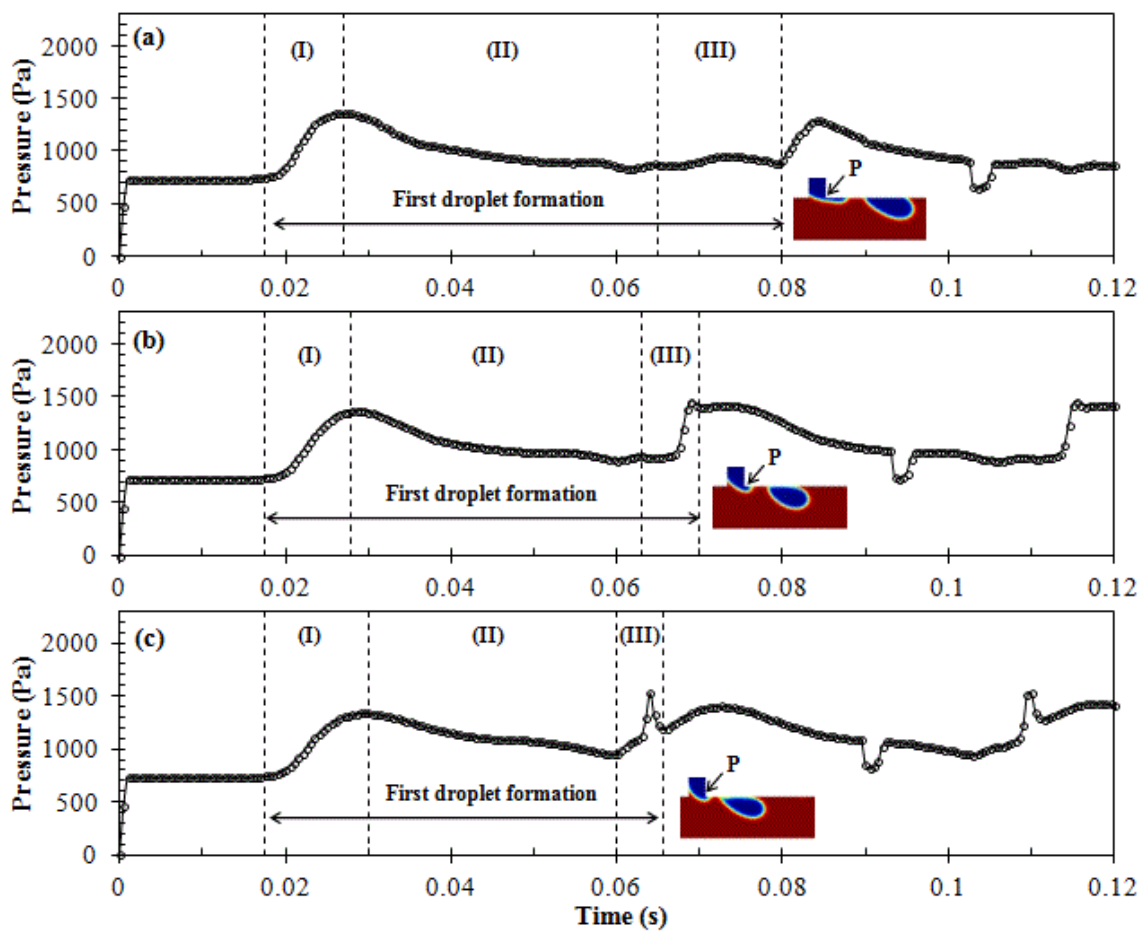

Figure 21: Pressure profile of $0.20 \mathrm{wt} \% \mathrm{CMC}$ droplets breakup for different wetting conditions: (a) $130^{\circ}$, (b) $150^{\circ}$, (c) $170^{\circ}$ (for system: $Q_{d}=0.10 \mathrm{ml} / \mathrm{hr} ; Q_{c}=2.00 \mathrm{ml} / \mathrm{hr}$ ). $P$ denotes the pressure at the corner of T-junction. The sharp peak in the pressure reflects the droplet breakup point as shown in the extracted images. The pressure peak becomes insignificant when the detachment point is located downstream of the junction.

\subsection{Effect of Interfacial Tension}

By considering the resisting force in the process of emulsification, it is generally accepted that interfacial tension is a significant element that markedly influences the evolution of the interface when droplets are forming ${ }^{56-57}$. In our simulations, six groups 
of cases with increasing values of interfacial tension from $0.010 \mathrm{~N} / \mathrm{m}$ to $0.035 \mathrm{~N} / \mathrm{m}$ were simulated. To obtain preferentially complete wetting of the continuous phase, a contact angle $\theta$ of $180^{\circ}$ was employed. Figure 22 illustrates the interfacial tension effect on the droplet diameter. The simulation predicts that the SCMC droplet diameter increases when the interfacial tension is increased, which is in agreement with the numerical results from Bashir et al. (2011) and Peng et al. (2011). The larger interfacial tension gives rise to a stronger driving force that attempts to minimize the surface area, ultimately inhibiting the break-up process. A larger interfacial force tends to delay the droplet formation process and resists the detachment of the droplets at higher surface tension (see equation 16). Consequently, a larger droplet diameter is required to balance the cross-flow drag force and the interfacial tension. This is illustrated in Figure 23, which shows a sequence of snapshots of droplet formation with the interfacial tension of $0.010 \mathrm{~N} / \mathrm{m}, 0.020 \mathrm{~N} / \mathrm{m}$, and $0.035 \mathrm{~N} / \mathrm{m}$ for 0.20 wt\% concentration of SCMC.

According to Laplace's law, the interfacial tension forces necessitate a pressure drop across the interface to maintain the interface at equilibrium against collapse. As described by the Young-Laplace law (Adamson and Gast, 1997), the pressure difference across a fluid interface is directly proportional to the interfacial tension and inversely proportional to the radius of curvature. Therefore, the higher the surface tension, the larger the required pressure difference across the interface. Figure 24 illustrates the plot of pressure profile for the $0.04 \mathrm{wt} \%$ SCMC solution at the corner of the T-junction at different interfacial tension. Here, higher interfacial tension leads to a greater pressure peak when the droplets break-up. A study made by Bashir et al. (2011) has reported that the tension across the interface of two phases may not be sufficient to prevent filament formation at lower interfacial tensions. 


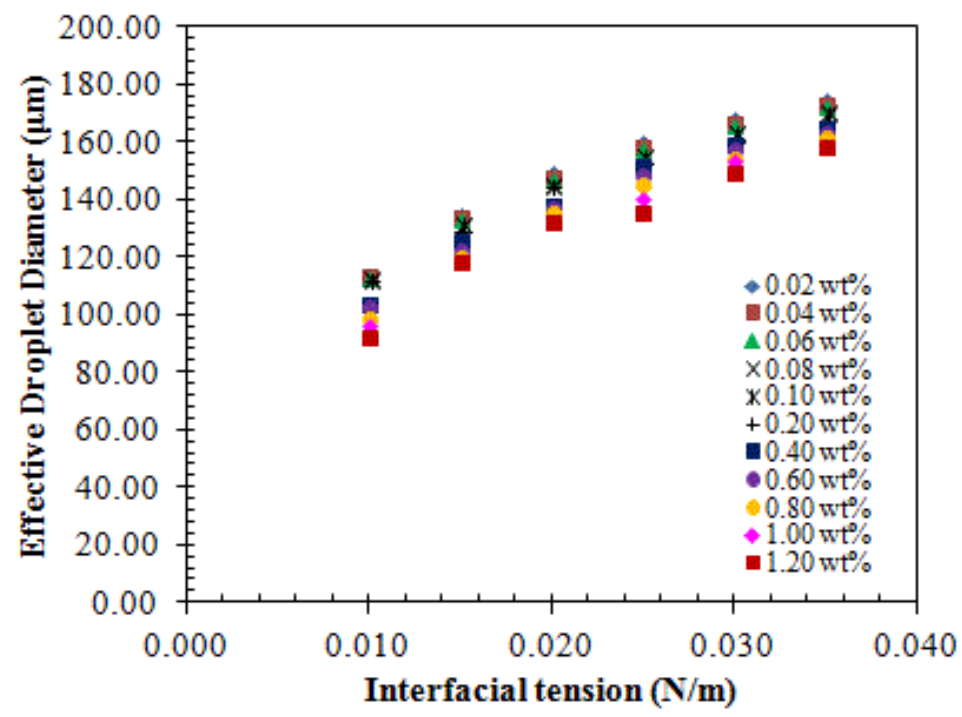

817

Figure 22: Effect of interfacial tension on the effective droplet diameter of SCMC solutions (for system: $Q_{d}=0.10 \mathrm{ml} / \mathrm{hr} ; Q_{c}=2.00 \mathrm{ml} / \mathrm{hr}$ ).

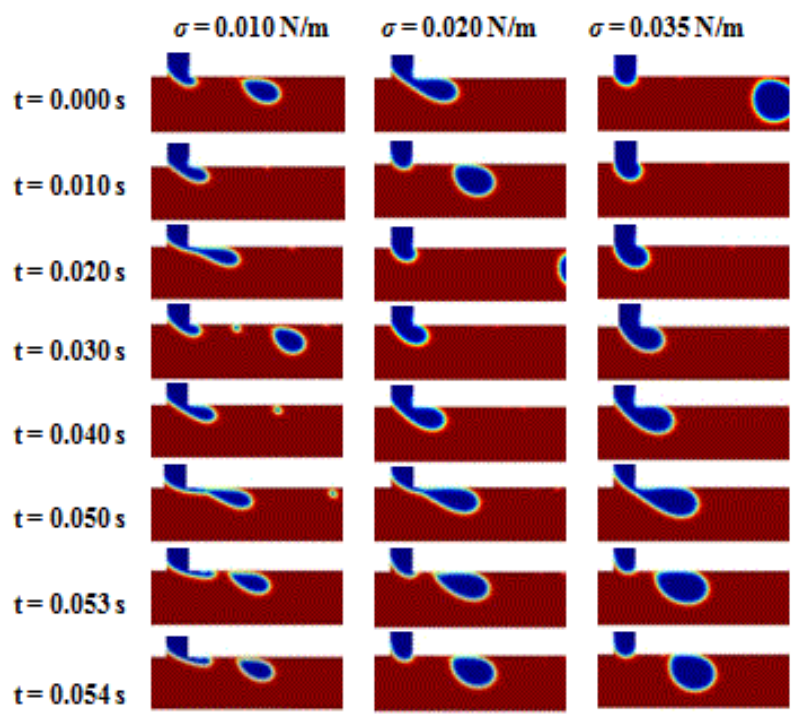

Figure 23: Snapshots of two-dimensional (2D) simulations of the effect of interfacial tension on $0.20 \mathrm{wt} \%$ SCMC-in-olive oil droplet breakup: (a) $\sigma=0.010 \mathrm{~N} / \mathrm{m}$, (b) $\sigma=$ $0.020 \mathrm{~N} / \mathrm{m}$, (c) $\sigma=0.035 \mathrm{~N} / \mathrm{m}$ (for system: $Q_{d}=0.10 \mathrm{ml} / \mathrm{hr} ; Q_{c}=2.00 \mathrm{ml} / \mathrm{hr}$ ). 

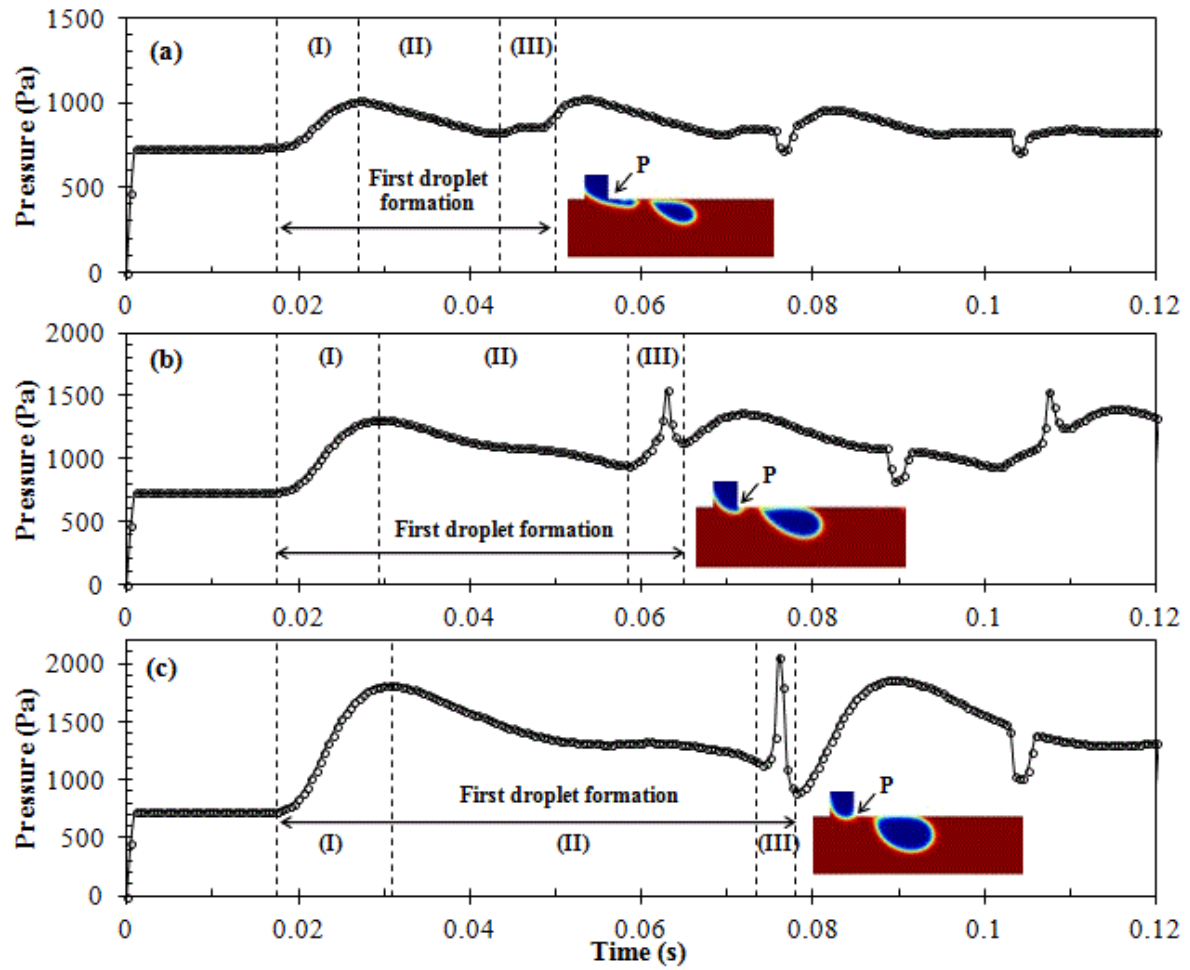

Figure 24: Pressure profile of during $0.20 w t \%$ SCMC droplet breakup for different interfacial tensions: (a) $\sigma=0.010 \mathrm{~N} / \mathrm{m}$, (b) $\sigma=0.020 \mathrm{~N} / \mathrm{m}$, (c) $\sigma=0.035 \mathrm{~N} / \mathrm{m}$ (for system: $Q_{d}=0.10 \mathrm{ml} / \mathrm{hr} ; Q_{c}=2.00 \mathrm{ml} / \mathrm{hr}$ ). $P$ denotes the pressure at the corner of Tjunction. The sharp peak with given data values reflects the droplet breakup point as shown in the extracted images. The pressure peak becomes insignificant when the detachment point is located downstream of the junction.

\section{Conclusions}

Emulsifying non-Newtonian solution in microfluidics in a controllable manner is one of the most difficult and least understood emulsification problems. The droplet formation of SCMC droplets in a Newtonian continuum was investigated for different flow conditions such as the volumetric flow rates of the continuous and dispersed phases, SCMC polymer concentration, continuous phase viscosity, interfacial tension, and contact angle. With thorough parametric studies, the evolution of SCMC droplet diameter and pressure distribution as function of these controlling parameters was elaborated. 
We varied the SCMC concentration, which modified the rheology of the disperse phase, increasing the viscosity and degree of shear thinning with increased SCMC concentration. We investigated the effect on droplet size. The strongest effects on the droplet size come from the continuous phase properties and flow rate, the interfacial tension and the contact angle. However, the disperse phase rate and rheology has a smaller, but still important effect. The effect of the disperse phase rheology is manifest in the viscosity ratio, which influences the mechanism by controlling the appearance and length of the downstream filament and has a direct effect on the droplet diameter. Diameters generally get smaller with increased SCMC concentration. Differences between the SCMC fluids become smaller with increased flow rates because stronger shear thinning in the more concentrated fluids narrows the difference in effective viscosity at higher flow rates.

All of these effects can be explained qualitatively by the idea that droplets detach when the drag on the droplet from the continuous phase balances the interfacial force that joins the droplet to the bulk region of disperse phase. Increasing the continuous phase viscosity or flow rate increase the drag force. Conversely, increasing the interfacial tension increases the force resisting detachment. The effect of the disperse phase viscosity is less direct. As the disperse phase viscosity decreases, internal flows within the droplet become possible and these lower the drag on the droplet. This explains the direction of the changes in droplet size with $\eta_{d}$. However, based on the modelling of Husny and Cooper-White ${ }^{11}$, the effect is expected to be fairly modest and may not fully explain the changes in droplet size seen in our simulations. For example figure 8 shows a fairly marked drop in the diameter between de-ionised water and $0.04 \mathrm{wt} \%$ SCMC solution. This may be because, even for Newtonian fluids, the full effect of is not correctly captured, perhaps because the walls interrupt the flow past the droplet in ways that are not included in the model of Husny and CooperWhite. Alternatively there may be qualitatively new effects that are introduced by shear thinning. Candidates for these non-Newtonian flow effects include the following. We assumed that the effective viscosity inside the droplet is determined by the wall shear rate in the continuous phase. However the internal flow in the droplet may be lower than this, meaning that the true $\eta_{d}$ is lower than our estimate, which would lead to smaller droplets. Secondly, shear thinning may drastically change the nature of the internal flow inside the droplet, leading to quantitatively different effects on the drag. 
879 For example, it is likely that a shear thinning fluid can localise the shear inside the 880 droplet to the region close to the droplet interface, in an effect similar to plug-flow in 881 a channel. This would suppress most of the internal flow and give greater drag and 882 hence smaller droplets.

883 Finally, we contrasted our finding with the experiments of Husny and Cooper-White 884 (2006), who studied Boger fluids. By varying the elasticity at fixed $\eta_{d}$, Husny and 885 Cooper-White (2006) changed the formation mechanism with negligible effect on the 886 droplet diameter. In contrast, by varying the viscosity and shear thinning of the 887 disperse phase, without elasticity, we showed a direct influence on the droplet size. 888 The combination of these two studies shows the potential to tailor the droplet size 889 and formation mechanism by controlling the shear-thinning and elasticity through 890 choice of polymer concentration and molecular weight.

\section{Acknowledgement}

893 Voon-Long Wong was supported by the University of Nottingham's Malaysia Intercampus 894 Doctoral Award Scheme (MIDAS). Katerina Loizou was supported by the Dean of 895 Engineering Scholarship awarded by the Faculty of Engineering, The University of 896 Nottingham during the PhD research candidacy. Both authors would like to thank the 897 respective research grants for enabling this work.

898 Nomenclature

899 a Fitting parameter in Carreau-Yasuda model

$900 \quad d \quad$ Effective droplet diameter $(\mu \mathrm{m})$

$901 F_{s t} \quad$ Surface tension force acting on the interface $\left(\mathrm{N} / \mathrm{m}^{3}\right)$

$902 F_{D} \quad$ Cross flow drag force $\left(\mathrm{N} / \mathrm{m}^{3}\right)$

$903 \quad h \quad$ Depth of the channel $(\mu \mathrm{m})$

904 I Identity matrix

$905 k$ Curvature of fluid-fluid interface 
906 n Power-law exponent for Carreau-Yasuda Model

$907 \mathbf{n}\ulcorner\quad$ Unit normal vector at the interface

$908 p$ Pressure $\left(\mathrm{N} / \mathrm{m}^{2}\right)$

$909 Q \quad$ Flow rate ratio

$910 Q_{c} \quad$ Flow rate of the continuous phase $(\mathrm{ml} / \mathrm{hr})$

$911 Q_{d} \quad$ Flow rate of the dispersed phase $(\mathrm{ml} / \mathrm{hr})$

$912 t \quad$ Time-step (s)

$913 u \quad$ Velocity component in x-direction $(\mathrm{m} / \mathrm{s})$

$914 \quad U \quad$ Maximum fluid velocity $(\mathrm{m} / \mathrm{s})$

$915 u \cdot \mathbf{n}$ Velocity component normal to the domain boundary $(\mathrm{m} / \mathrm{s})$

$916 u_{0} \quad$ Initial velocity $(\mathrm{m} / \mathrm{s})$

$917 \quad V \quad$ Velocity component in y-direction (y-direction) $(\mathrm{m} / \mathrm{s})$

$918 \quad V^{*} \quad$ Continuous phase velocity $(\mathrm{m} / \mathrm{s})$

919 u Velocity field

$920 \quad W_{c} \quad$ Width of the continuous phase channel $(\mu \mathrm{m})$

$921 W_{d} \quad$ Width of the dispersed phase channel $(\mu \mathrm{m})$

$922 x \quad$ Longitudinal coordinates axes

$923 \quad X \quad$ Computational cell size $(\mathrm{m})$

$924 \quad y \quad$ Lateral coordinates axes

925

926 Greek Symbols

$927 \quad \eta \quad$ Dynamic viscosity of fluid (Pa.s)

$928 \quad \eta_{d} \quad$ Dynamic viscosity of the dispersed phase (Pa.s) 
$929 \eta_{c} \quad$ Dynamic viscosity of the continuous phase (Pa.s)

$930 \quad \eta_{0} \quad$ Zero shear viscosity (Pa.s)

$931 \quad \eta_{\infty} \quad$ Infinite shear viscosity (Pa.s)

$932 \quad \lambda_{\eta} \quad$ Viscosity ratio $\left(\eta_{d} / \eta_{c}\right)$

$933 \lambda_{c y}$ Relaxation Time in Carreau-Yasuda Model(s)

$934 \rho \quad$ Fluid density $\left(\mathrm{kg} / \mathrm{m}^{3}\right)$

$935 \quad T \quad$ Shear stress $(\mathrm{Pa})$

$936 \dot{\gamma} \quad$ Shear rate $(1 / \mathrm{s})$

$937 \quad \gamma \quad$ Reinitialization parameter $(\mathrm{m} / \mathrm{s})$

$938 \varepsilon \quad$ Thickness of the interface $(\mathrm{m})$

$939 \sigma \quad$ Interfacial tension $(\mathrm{mN} / \mathrm{m})$

$940 \phi \quad$ Level set function

$941 \delta_{s m}$ Dirac delta function concentrated at interface

$942 \Omega \quad$ Computational domain

$943 \partial \Omega \quad$ Domain boundary

945 References

Adamson AW, Gast AP. Physical Chemistry of Surfaces, Wiley: New York. Sixth 947 edition; 1997: 4-35.

Anna SL, Mayer HC. Microscale tip streaming in a microfluidic flow focusing device. $949 \quad$ Phys Fluids, 2006; 18: 121512.

950 Arratia PE, Cramer LA, Gollub JP, Durian DJ. The effects of polymer molecular weight 951 on filament thinning and drop breakup in microchannels. New J Phys. 2009; 11: $952115006(1-18)$. 
953 Bashir S, Rees JM, Zimmerman WB. Simulations of microfluidics droplet formation using the two-phase level set method. Chem Eng Sci. 2011; 66: 4733-4741.

Bonometti T, Magnaudet J. An interface-capturing method for incompressible twophase flows. Validation and application to bubble dynamics. Int J Multiphase Flow, 2007; 33: 109-133.

Carreau P. Rheological equations from molecular network theories. T Soc Rheol. 1972; 16: 99.

Chhabra RP, Richardson JF. Non-Newtonian flow and applied rheology, $2^{\text {nd }}$ ed. Oxford: Butterworth-Heinemann; 2008.

Christopher GF, Bergstein J, End NB, Poon M, Nguyen C, Anna SL. Coalescence and splitting of confined droplets at microfluidic junctions. Lab Chip. 2009; 9: 11021109.

Chung CK, Hulsen MA, Kim JM, Ahn KH, Lee SJ. Numerical study on the effect of viscoelasticity on drop deformation in simple shear and 5:1:5 planar contraction/expansion microchannel. J Non-Newton Fluid Mech. 2008; 155:80-93.

Cramer C, Fischer P, Windhab EJ. Drop formation in a co-flowing ambient fluid. Chem Eng Sci. 2004; 59: 3045-3058.

Davidson MR, Cooper-White JJ, Tirtaatmadja V. Shear-thinning drop formation. ANZIAM J. 2004; 450: 405-418.

Davidson MR, Cooper-White JJ. Pendant drop formation of shear-thinning and yield stress fluids. Appl Math Model. 2006; 30: 1392-1405.

DeMenech M, Garstecki P, Jousse F, Stone HA. Transition from squeezing to dripping in a microfluidic T-shaped junction. J Fluid Mech. 2008; 595:141-161.

Garstecki P, Fuerstman MJ, Stone HA, Whitesides GM. Formation of droplets and bubbles in a microfluidic T-junction-scaling and mechanism of breakup. Lab Chip. 2006; 6:437-446. 
Garstecki P, Stone HA, Whitesides GM. Mechanism for Flow-Rate Controlled Breakup in Confined Geometries: A Route to Monodisperse Emulsions. Phys Rev Lett. 2005; 94: 164501(1-4).

Glawdel T, Elbuken C, Ren CL. Droplet formation in microfluidic T-junction generators operating in the transitional regime. Il. Modelling. Phys Rev, 2012; 85: 016323 (112).

Gu ZP, Liow JL. Microdroplet formation in a T-junction with xanthan gum solutions. Chemeca: Australasian Conference on Chemical Engineering; 2011.

Hong JS, Cooper-White J. Drop formation of Carbopol dispersions displaying yield stress, shear thinning and elastic properties in a flow-focusing microfluidic channel. Korea-Aust Rheol J. 2009; 21: 269-280.

Hou TY, Lowengrub JS, Shelley MJ. Boundary integral methods for multicomponent fluids and multiphase materials. J Comput Phys, 2001; 169: 302-362.

Husny J, Cooper-White JJ. The effect of elasticity on drop creation in T-shaped microchannels. J Non-Newton Fluid Mech. 2006; 137:121-136.

Husny J, Jin HY, Harvey E, Cooper-White JJ. Dynamics of Shear-Induced Drop Formation in T-Shaped Microchannels. Seventh International Conference on Miniaturized Chemical and Biochemical Analysts Systems. 2003; 1: 113-116.

Kashid MN, Renken A., Kiwi-Minsker L. CFD modelling of liquid-liquid multiphase microstructured reactor: slug flow generation. Chem Eng Res Des. 2010; 88; 362368.

Kobayashi I, Mukataka S, Nakajima M. High Performance Flow Focusing Geometry For Spontaneous Generation of Monodispersed Droplets. Ind Eng Chem Res. 2005; 44: 5852-5856.

Kobayashi I, Nakajima M, Chun K, Kikuchi Y, Fukita H. Silicon Array of Elongated Through-Holes for Monodisperse Emulsion Droplets. AIChE J. 2002; 48: 16391644.

Kumacheva E, Garstecki P. Microfluidic Reactors for Polymer Particles, Wiley: New York; 2011. 
Liu H, Zhang Y. Droplet formation in T-shaped microfluidic junction. J Appl Phys. 2009; 106: 034906 (1-8).

Loizou K, Wong VL, Thielemans W, Hewakandamby BN. Effect of Fluid Properties on Droplet Generation in a Microfluidic T-junction. Proceedings of the ASME $4^{\text {th }}$ Joint US-European Fluids Engineering Division Summer Meeting, Chicago, Illinois; 3-8 August 2014; 9pp.

Nisisako T, Torii T, Higuchi T. Droplet formation in a microchannel network. Lab Chip. 2002; 2(1): 24-26.

Nunes JK, Tsai SSH, Wan J, Stone HA. Dripping and jetting in microfluidic multiphase flows applied to particle and fibre synthesis. J Phys D: Appl Phys. 2013; 46: 114002 (1-20).

Olsson E, Kreiss G, Zahedi S. A conservative level set method for two phase flow II. J Comput Phys. 2007; 225(1): 785-807.

Olsson E, Kreiss G. A conservative level set method for two phase flow. J Comput Phys. 2005; 210: 225-246.

Osher S, Sethian JA. Fronts propagating with curvature-dependent speed: algorithms based on Hamilton-Jacobi formulations. J Comput Phys. 1988; 79: 12-49.

Peng L, Yang M, Guo SS, Liu W, Zhao XZ. The effect of interfacial tension on droplet formation in flow focusing microfluidic device. Biomed Microdevices. 2011; 13: 559-564.

Pipe CJ, McKinley GH. Microfluidic rheometry. Mech Res Commun. 2009; 36: 110120.

Qiu DM, Silva L, Tonkovich AL, Arora R. Micro-droplet formation in non-Newtonian fluid in a microchannel. Microfluid Nanofluid. 2010; 8: 531-548.

Rider WJ, Kothe DB. Reconstructing volume tracking. J Comput Phys, 1998; 141: $112-152$.

Rosengarten G, Harvie DJE, Cooper-White J. Contact angle effects on microdroplet deformation using CFD. Appl Math Model. 2006; 30: 1033-1042. 
Sang L, Hong YP, Wang FJ. Investigation of viscosity effect on droplet formation in Tshaped microchannels by numerical and analytical methods. Microfluid Nanofluid. 2009; 6: 621-635.

Schneider T, Burnham DR, VanOrden J, Chiu DT. Systematic investigation of droplet generation at T-junctions. Lab Chip. 2011; 11; 2055-2059.

Sethian JA. Level Set Methods and Fast Marching Methods: Evolving Interfaces in Computational Geometry, Fluid Mechanics, Computer Vision and Materials Sciences. Cambridge, MA: Cambridge University Press Second Edition; 1999.

Squires TM, Quake SR. Microfluidics: Fluid physics at the nanoliter scale. Rev Mod Phys. 2005; 77: 977-1024.

Steinhaus B, Shen AQ, Sureshkumar R. Dynamics of viscoelastic fluid filaments in microfluidic devices. Phys Fluids. 2007; 18: 073103(1-13).

Stone HA, Stroock AD, Ajdari A. Engineering flows in small devices: microfluidics toward lab-on-a-chip. Annu Rev Fluid Mech. 2004; 36: 381-411.

Sugiura S, Nakajima M, Iwamoto S, Seki M. Interfacial Tension Driven Monodispersed Droplet Formation from microfabricated channel array. Langmuir, 2001; 17: 5562-5566.

Takada N, Misawa M, Tomiyama A, Fujiwara S. Numerical simulation of two- and three-dimensional two phase fluid motion by Lattice Boltzmann method. Comput Phys Commun, 2000; 129: 233- 246.

Tan SH, Murshed SMS, Nguyen NT, Wong TN, Yobas L. Thermally controlled droplet formation in flow focusing geometry: formation regimes and effect of nanoparticle suspension. J Phys D: Appl Phys. 2008; 41: 165501. doi:10.1088/00223727/41/16/165501

Thorsen T, Roberts RW, Arnold FH, Quake SR. Dynamic Pattern Formation in a Vesicle- Generating Microfluidic Device. Phys Rev Lett. 2001; 86(16): 4163-4166.

Tice JD, Lyon AD, Ismagilov RF. Effects of viscosity on droplet formation and mixing in microfluidic channels. Anal Chim Acta. 2004; 507: 73-77. 
1064 Tryggvason G, Bunner B, Esmaeeli A, et al. A front-tracking method for the 1065 computations of multiphase flow. J Comput Phys, 2001; 169(2): 708-759.

1066 Utada AS, Fernandez-Nieves A, Stone HA, Weitz DA. Dripping to jetting transitions in co-flowing liquid streams. Phys Rev Lett. 2007; 99:094502.

1068 Van der Zwan E, Schroen K, Boom R. A Geometric Model for the Dynamics of 1069 Microchannel Emulsification. Langmuir. 2009; 25(13): 7320-7327.

1070 Wehking JD, Gabany M, Chew L, Kumar R. Effects of viscosity, interfacial tension, 1071 and flow geometry on droplet formation in a microfluidic T-junction. Microfluid $1072 \quad$ Nanofluid. 2014; 16: 441-453.

Xu JH, Li SW, Tan J, Wang YJ, Luo, GS. Preparation of Highly Monodisperse Droplet 1074 in a T-Junction Microfluidic Device. AIChE J. 2008; 52: 3005-3010.

Yasuda RAK, Cohen R. Shear flow properties of concentrated-solutions of linear and star branched polystyrenes. Rheol Acta. 1981; 20: 163.

Yeom S, Lee SY. Size prediction of drops formed by dripping at a micro T-junction in liquid-liquid mixing. Exp Therm Fluid Sci. 2011; 35(2): 387-394.

1079 Zhang X, Basaran OA. An experimental study of dynamics of drop formation. Phys 1080 Fluids. 1995; 7: 1184-1203. 